\title{
Inverse problem for a coupling model of reaction-diffusion and ordinary differential equations systems. Application to an epidemiological model.
}

\author{
Nathalie Verdière ${ }^{\mathrm{a}, *}$, David Manceau ${ }^{\mathrm{a}}$, Shousheng $\mathrm{Zhu}^{\mathrm{b}}$, Lilianne Denis-Vidal $^{\mathrm{b}}$ \\ ${ }^{a}$ Normandie Univ, UNIHAVRE, LMAH, FR-CNRS-3335, ISCN, 76600 Le Havre, France \\ ${ }^{b}$ Applied Mathematics Laboratory of Compiègne (LMAC), University of Technology of \\ Compiègne, France
}

\begin{abstract}
This paper investigates an identifiability method for a class of systems of reaction diffusion equations in the $L^{2}$ framework. This class is composed of a master system of ordinary differential equations coupled with a slave system of diffusion equations. It can model two populations, the second one being diffusive contrary to the first one. The identifiability method is based on an elimination procedure providing relations called input-output polynomials and linking the unknown parameters, the inputs and the outputs of the model. These polynomials can also be used to estimate the parameters as shown in this article. To our best knowledge, such an identifiability method and a parameter estimation procedure have not yet been explored for such a system in the $L^{2}$ framework. This work is applied on an epidemiological model describing the propagation of the chikungunya in a local population.
\end{abstract}

Keywords: Identifiability; PDEs and ODEs systems; Parameter estimation; Epidemiological models

\section{Introduction}

Many biological or physical processes are modeled using ordinary differential equations (ODE) or partial differential equations (PDE) to describe the (spatial) evolution over the time of quantities of interest. However, they depend on

\footnotetext{
${ }^{*}$ Corresponding author

Email address: nathalie.verdiere@univ-lehavre.fr (Nathalie Verdière)
} 
many parameters that need to be estimated from the measurements of the system to make the mathematical model useful. This kind of problem, called inverse problem, necessitates, in general, an identifiability study. An identifiability study ensures that the parameters of interest can be uniquely inferred from given measurements. Consequently, it is a necessary prerequisite before putting in place parameter identification procedures, otherwise, the latter can fail or give not realistic parameter values. This theoretical work is done in the 'best-case' that is from noise-free data.

Identifiability study for ODEs systems is well known and several methods exist in the literature for linear or nonlinear systems (see for example [11, 14, 16, $18,26,30,31,33]$ and the references therein). In the case of PDEs systems (or equations), most of the work on inverse problems concerns a source problem (see $[2,7,10,13,23,36]$ ) or a Calderòn's problem (see the original paper [5] and the review on the subject [29]). This kind of inverse problems consists in identifying a source term, or to determine a space variable parameter, from boundary measurements.

The inverse problem studied in this paper for PDEs systems is of different type to the one handled in the previous references. Indeed, the difference comes from the knowledge of one (or more) of its solutions (on all the domain or just a part of it). Even if this assumption seems to be less considered, some works can be found in this direction. An identifiability study has been proposed in [34] (see also reference [6] for a more recent reference) for a SIS (Susceptible - Infectious Susceptible) epidemic reaction-diffusion model using an optimal control method. In [24], using input-output relationships, identifiability of a system of nonlinear integro-partial differential equations of transport type was proposed. An approach based on semigroup theory to identify the mortality rate in an age-structured population dynamics model have been proposed in [25]. In the case of coupled ODEs and PDEs systems, a study of identifiability have been done in [15] by means of Carleman-type estimate, and in [28] using the differential algebra approach where the PDE model is an age-since-infection-structured population dynamics model.

In this paper, we propose an identifiability method, using the differential algebra approach, for some coupled nonlinear ODEs and reaction-diffusion systems composed of rational functions. This assumption is not so restrictive since by change of variables, lots of systems can be rewritten as rational ones. From the elimination theory developed in the case of ODEs systems, some input-output (IO) polynomials are obtained. The method that we propose is based on these specific relations permitting in the same way to propose a numerical procedure giving a first estimate of the parameters by direct measurements without any $a$ 
priori knowledge. To our best knowledge, such an identifiability method has not yet been studied for a coupled nonlinear ODEs and reaction-diffusion systems in the $L^{2}$ framework.

For the application of the method, we propose to consider the model given in [20] and describing the transmission of the chikungunya to the human population by the mosquito vector, the Aedes Albopictus. This disease is still current since this vector has developed capabilities to adapt to non tropical regions and a genetic mutation has reduced the delay between the contamination of the host and its propagation to humans. In 2014, several cases have been reported in the South of America, the Caraibes and central America. In August 2017, it is the France and Italy that have been concerned. In [20], the virus transmission to the human population has been modeled by a SI-SIR (Susceptible - Infectious and Susceptible - Infectious - Recovered) model taking into account the mosquito biological life cycle. An identifiability study of this model has been done in [27]. But this ODEs system does not consider an important factor favoring the re-emergence of diseases, that is the spatial mobility of humans. In order to consider this spatial mobility, a metapopulation model has been proposed in [21]. This spatio-temporal model can be seen as a discrete model in space. In this paper, we model human mobility by a diffusion term in the same way as [22] or [1, 17]. In [37], an identifiability study was proposed on this model in the continuous framework, that is with continuous initial datum. In this paper, we revisit this work with less assumptions on the regularity of the solutions. More precisely, we work in the $L^{2}$ framework.

The paper is structured as follows. In section 2, we present the coupling model which is considered in the sequel. Section 3 is devoted to general identifiability results based on the elimination theory and their applications on the coupling model. In section 4, the theoretical part is applied on a chikungunya epidemiological model and a parameter estimation method based on the input-output polynomials is proposed in section 5 to estimate two of the model parameters. Section 6 concludes the paper.

\section{Coupling model}

\subsection{Notations}

The notations are the following:

- $\Omega$ is a bounded domain of $\mathbb{R}^{n}$ with $C^{2}$-boundary. 
- $C_{l}([0, \infty) ; C(\bar{\Omega}))$ denotes the space of piecewise continuous functions from $[0, \infty)$ to $C(\bar{\Omega})$ and $C_{l}^{1}([0, \infty) ; C(\bar{\Omega}))$ the space of piecewise differentiable functions from $[0, \infty)$ to $C(\bar{\Omega})$.

- Given a local Lebesgue-integrable function $F, T_{F}$ defines its regular distribution.

- $H_{N}^{2}(\Omega):=\left\{v \in H^{2}(\Omega) \mid \frac{\partial v}{\partial \nu}=0\right.$ on $\left.\partial \Omega\right\}$, where $\nu$ is the outward unit normal of $\partial \Omega$.

- For any function $f \in L^{1}(\Omega)$, we set $\langle f\rangle:=\frac{1}{|\Omega|} \int_{\Omega} f(x) d x$.

\subsection{Coupling model}

The model we investigate in this paper is a master-slave model of two species where the slave one diffuses, contrary to the master one. The master model is a system of classical ordinary differential equations defined on the space $C(\bar{\Omega})$ given by:

$$
\left\{\begin{aligned}
\partial_{t} \zeta & =F(t, \zeta), \text { in }(0, \infty), \\
\zeta(0) & =\zeta_{0} .
\end{aligned}\right.
$$

where $\zeta_{0} \in C(\bar{\Omega})$ and $F: \mathbb{R} \times \mathbb{R}^{p} \rightarrow \mathbb{R}^{p}$ is piecewise continuous with respect to the first component and uniformly Lipschitz continuous with respect to the second component. Then, from the Cauchy-Lipschitz theorem, there exists a unique piecewise differentiable solution $\zeta$ from any time interval of existence to $C(\bar{\Omega})$, i.e. $\zeta \in C_{l}^{1}([0, \infty) ; C(\bar{\Omega}))$.

The slave model is a system of reaction-diffusion equations defined on the Banach space $X:=C\left(\Omega ; \mathbb{R}^{q_{0}}\right) \times L^{2}\left(\Omega ; \mathbb{R}^{q_{1}}\right)$ and which depends on the solution $\zeta$ of the master model:

$$
\left\{\begin{aligned}
\partial_{t} U+B U & =G(t, \zeta, U), \quad \text { in }(0, \infty), \\
U(0) & =U_{0},
\end{aligned}\right.
$$

where $U_{0} \in X$ and the diagonal operator $B:=\operatorname{diag}\left(B_{i}\right)$ on $X$ satisfies

- for all $i \in\left\{1, \ldots, q_{0}\right\}, B_{i}:=I$,

- for all $i \in\left\{q_{0}+1, \ldots, q\right\}$ (where $q:=q_{0}+q_{1}$ ), $B_{i}:=d_{i} A+b_{i}$ with $d_{i}, b_{i}>0$ and $A$ is the realization of the Laplacian in $L^{2}(\Omega)$ with Neumann homogeneous boundary condition on $\partial \Omega$. 
In other words, (2) is composed of $q_{0}$ ODEs and $q_{1}$ PDEs which are all together coupled.

Moreover, $G(t, \zeta, \cdot)$ is a non-linear operator from $D\left(B^{\eta}\right)$ into $X$ (see (24)), where $0<\eta<1$, which satisfies the following Lipschitz type condition: there exists an increasing function $\phi$ such that, for any compact time interval $I$ where $\zeta$ is differentiable and for all $(t, U),(s, V) \in I \times D\left(B^{\eta}\right)$, we have

$$
\begin{aligned}
& \|G(t, \zeta, U)-G(s, \zeta, V)\|_{X} \leq \phi\left(\|U\|_{X}+\|V\|_{X}\right) \\
& \quad \times\left[\left\|B^{\eta}(U-V)\right\|_{X}+\left(\left\|B^{\eta} U\right\|_{X}+\left\|B^{\eta} V\right\|_{X}+1\right)\left(|t-s|+\|U-V\|_{X}\right)\right],
\end{aligned}
$$

where $\|\cdot\|_{X}$ denotes the norm of $X$.

Then, a simple adaptation of Theorem 4.4 of [35] (p. 188, and p. 199 for the non autonomous case) implies that there exists $T_{U_{0}}>0$ such that (2) admits a unique local solution

$$
U \in C_{l}\left(\left[0, T_{U_{0}}\right] ; D(B)\right) \cap C_{l}^{1}\left(\left(0, T_{U_{0}}\right] ; X\right) .
$$

Note that $U$ is piecewise continuous and piecewise differentiable due to the dependence with respect to $\zeta$.

Moreover, if there exists $C_{U_{0}}>0$ such that the local solution $U$ satisfies:

$$
\forall t \in I, \quad\|U(t)\|_{2} \leq C_{U_{0}}
$$

for any compact time interval $I$ where $U$ is continuous with respect to the time variable, then $U$ is a global solution:

$$
U \in C_{l}([0, \infty) ; D(B)) \cap C_{l}^{1}((0, \infty) ; X) .
$$

Such systems depend on parameters not always directly accessible from measurements or by mean of a costly and experimental scheme. However, they must be estimated to make the model usable. Several numerical procedures, based on measurements, can be found in the literature. They carried out by optimizing some criterion function over the parameter space. However, an identifiability study should be done since it is a pre-condition for safely running a parameter estimation algorithm. Indeed, it will ensure that the algorithm will not converge towards an unrealistic solution.

More precisely, we introduce a parameter vector function $\theta:=\left(\theta_{a}, \theta_{b}\right) \in L^{\infty}\left(\Omega ; \mathcal{U}_{p}\right)$, where $\mathcal{U}_{p}$ is an open subset of $\mathbb{R}^{p}$, and some corresponding model outputs $y(t, \theta):=$ $\left(y_{a}\left(t, \theta_{a}\right), y_{b}\left(t, \theta_{b}\right)\right)$. Thus, we rewrite models (1) and (2) in the following form: 


$$
\left(\Gamma_{a}\right)\left\{\begin{aligned}
\partial_{t} \zeta\left(t, \theta_{a}\right) & =f\left(t, \zeta, \theta_{a}\right), & & t \in[0, \infty), \\
y_{a}\left(t, \theta_{a}\right) & =h_{a}(\zeta), & & t \in[0, \infty),
\end{aligned}\right.
$$

completed with $\zeta\left(t=0, \theta_{a}\right)=\zeta_{0}$, and

$$
\left(\Gamma_{b}\right)\left\{\begin{aligned}
\partial_{t} U\left(t, \theta_{b}\right)+B\left(\theta_{b}\right) U\left(t, \theta_{b}\right) & =g\left(\zeta, U, \theta_{b}\right), & & t \in[0, \infty), \\
y_{b}\left(t, \theta_{b}\right) & =h_{b}(\zeta, U), & & t \in[0, \infty),
\end{aligned}\right.
$$

completed with $U\left(t=0, \theta_{b}\right)=U_{0}$. The notations are the following:

- $f:=\left(f_{1}, \ldots, f_{p}\right)$ where $f_{i}, 1 \leq i \leq p$, is a rational function with respect to $\zeta$ and $\theta_{a}$,

- $g:=\left(g_{1}, \ldots, g_{q}\right)$ where $g_{i}, 1 \leq i \leq q$, is a rational function with respect to $\zeta, U$ and $\theta_{b}$,

- $h_{a}:=\left(h_{a, 1}, \ldots, h_{a, m_{a}}\right)\left(\right.$ resp. $\left.h_{b}:=\left(h_{b, 1}, \ldots, h_{b, m_{b}}\right)\right)$ where $h_{a, i}, 1 \leq i \leq$ $m_{a}$ (resp. $h_{b, i}, 1 \leq i \leq m_{b}$ ), are rational functions with respect to $\zeta$ (resp. $\zeta$ and $U)$.

Afterwards, $\left(\Gamma_{\theta}\right)$ will design either system $\left(\Gamma_{a}\right)$ or $\left(\Gamma_{b}\right)$.

\section{Identifiability}

\subsection{General Case}

The identifiability definition considered in this paper is the following:

Definition 3.1. We say that the model $\left(\Gamma_{\theta}\right)$ is identifiable with respect to $\theta$ if there exists a time $t_{1}>0$ and a subset $\bar{\Omega}$ of $\Omega$ such that, for all $\theta, \tilde{\theta} \in L^{\infty}\left(\Omega ; \mathcal{U}_{p}\right)$, we have

$$
\forall(t, \mathbf{x}) \in\left[0, t_{1}\right] \times \bar{\Omega}, \quad y(t, \theta)(\mathbf{x})=y(t, \tilde{\theta})(\mathbf{x}) \Longrightarrow \theta=\tilde{\theta} .
$$

Definition 3.1 states that there exists a time interval and a space domain such that two distinct parameters will not generate identical model outputs. This definition can be easily extended to piecewise continuous outputs by considering the model's identifiability on each subinterval on which the outputs are defined.

We propose to extend an identifiability method based on the differential algebra theory applied to ODEs with constant parameters $[3,4,8,9]$. This method is 
based on some differential polynomials obtained owing to an elimination procedure. The latter consists in eliminating unobservable variables for the benefit of the outputs and the parameters of the model.

Differential algebra theory provides an automatic elimination tool for performing specific relations depending only on the inputs, the outputs and the parameters of the system. These relations are considered as polynomials whose indeterminates are the inputs and the outputs of the model and are also called input-ouptut polynomials. In certain cases, the elimination procedure can be done by hand, however, dedicated packages are available, for example DifferentialAlgebra implemented in Maple [3, 4]. This method necessitates most of the times derivation operations and, in the case of the use of a dedicated software, the elimination is done in a formal way, that is, without any consideration of the solution space. The relations can also not be defined with respect to the solution regularity of the model. Nevertheless, new well-defined relations can be obtained in considering the previous relations in the distribution sense or from the mean value, so that an identifiability result can be deduced on the studied system.

Suppose that, from an elimination procedure considering only the derivation with respect to the time, the differential polynomials $P_{i}\left(y_{\theta}, \theta, u\right)$ depending on $\theta$, $y_{\theta}$ and the input function $u$ of the model have been obtained. The number of these polynomials is the number of model outputs according to [8]. To simplify the notations, we consider afterwards that we have only one output, that is one differential relation $P\left(y_{\theta}, \theta, u\right)$ (which correspond to the case of the application of section 4). This differential polynomial can be expressed as (see [8]):

$$
P\left(y_{\theta}, \theta, u\right)=m_{0}\left(y_{\theta}, u\right)+\sum_{k=1}^{n} \gamma_{k}(\theta) m_{k}\left(y_{\theta}, u\right) \quad \text { and } \quad P\left(y_{\theta}, \theta, u\right)=0
$$

where $\left(\gamma_{k}(\theta)\right)_{1 \leq k \leq n}\left(\gamma_{\alpha} \neq \gamma_{\beta}\right.$ if $\alpha \neq \beta$ and $\left.\alpha, \beta \in\{1, \ldots, n\}\right)$ are rational in $\theta,\left(m_{k}\left(y_{\theta}, u\right)\right)_{0 \leq k \leq n}$ are differential polynomials with respect to $y_{\theta}$ and $u$, and $m_{0} \neq 0$.

\section{Remark 3.1.}

1. In the present case, a differential polynomial with respect to $y_{\theta}$ means a multivariate polynomial of the variables $y_{\theta}, \partial_{t} y_{\theta}, \partial_{t}^{2} y_{\theta}, \ldots$

2. The denominators of $\gamma_{k}(\theta)$ are supposed not to vanish.

3. For system $\left(\Gamma_{b}\right)$, the function $\zeta$ can be considered as an input function. 
4. By using Rosenfeld-Groebner algorithm, one can obtain a relation of the form (7) such that the $m_{k}\left(y_{\theta}, u\right)$ for $k=1, \ldots, n$ are linearly independent (see [12]).

The family $\left\{\gamma_{1}(\theta), \ldots, \gamma_{n}(\theta)\right\}$ is called the exhaustive summary of $P$ and the differential polynomial $P$ is called an input-output polynomial.

Example 3.1. We illustrate the notion of input-output polynomial on a simple problem. Consider an ODEs system given by

$$
\left\{\begin{array}{l}
\dot{x_{1}}=u \\
\dot{x_{2}}=x_{1}+k u
\end{array}\right.
$$

where $k \in \mathbb{R}$ is a parameter to identify $(\theta=k)$ and $u$ is an input function.

Suppose $u$ is $C^{1}$ and assume that $y:=x_{2}$ is observable. Then, $y$ is $C^{2}$ and we have

$$
\ddot{y}=\dot{x_{1}}+k \dot{u}=u+k \dot{u} .
$$

Thus, we deduce that we have an input-output polynomial $P$ such that $P(y, k, u)=$ 0 which is given by

$$
P(y, k, u):=\ddot{y}-u-k \dot{u} .
$$

Moreover, the exhaustive summary is simply $\{k\}$.

Note that we assumed that $u$ is $C^{1}$. We will see below how we can handle a more general case of an input function with less regularity.

Suppose that the elimination process respected the regularity of the solutions of the ODEs or the PDEs system. Then the following proposition gives a necessary condition to obtain the identifiability of the model from the differential polynomial (7).

Proposition 3.1. Assumed that the family $\left(m_{k}\left(y_{\theta}, u\right)\right)_{1 \leq k \leq n}$ given by (7) is linearly independent. If, for all $(\theta, \tilde{\theta}) \in L^{\infty}\left(\Omega, \mathcal{U}_{p}\right)^{2}$, we have

$$
\forall k \in\{1, \ldots, n\}, \quad \gamma_{k}(\theta)=\gamma_{k}(\tilde{\theta}) \Longrightarrow \theta=\tilde{\theta},
$$

then the model is identifiable with respect to $\theta$.

Proof 3.1. Let $(\theta, \tilde{\theta}) \in L^{\infty}\left(\Omega, \mathcal{U}_{p}\right)^{2}$ be such that $y_{\theta}(t, \theta)=y_{\theta}(t, \tilde{\theta})$. From (7), one gets:

$$
\sum_{k=1}^{n}\left(\gamma_{k}(\theta)-\gamma_{k}(\tilde{\theta})\right) m_{k}\left(y_{\theta}, u\right)=0
$$


According to the linear independence of the family $\left(m_{k}\left(y_{\theta}, u\right)\right)_{1 \leq k \leq n}$, relation (9) implies

$$
\forall k \in\{1, \ldots, n\}, \quad \gamma_{k}(\theta)=\gamma_{k}(\tilde{\theta}) .
$$

Thus, by assumption, $\theta=\tilde{\theta}$.

By using dedicated software programs, relation (7) is obtained in a formal way by differentiation with respect to the time and combination of the equations of the initial system. Its derivative degrees may be too high with respect to the solution regularity.

Assume that, in (7), the $m_{k}\left(y_{\theta}, u\right), k=0, \ldots, n$, are well defined almost everywhere and are locally Lebesgue integrable. Then, one can replace the not well defined $m_{k}\left(y_{\theta}, u\right)$ by their distributions $T_{m_{k}\left(y_{\theta}, u\right)}$. Thus, we obtain a new relation of the form

$$
T_{m_{0}\left(y_{\theta}, u\right)}+\sum_{k=1}^{n} \gamma_{k}(\theta) T_{m_{k}\left(y_{\theta}, u\right)}=0 .
$$

In this case, similarly to Proposition 3.1, we have the following result in the distribution sense.

Proposition 3.2. Assumed that the family of distributions $\left(T_{m_{k}\left(y_{\theta}, u\right)}\right)_{1 \leq k \leq n}$ given by (10) is linearly independent. If, for all $(\theta, \tilde{\theta}) \in L^{\infty}\left(\Omega, \mathcal{U}_{p}\right)^{2}$, we have

$$
\forall k \in\{1, \ldots, n\}, \quad \gamma_{k}(\theta)=\gamma_{k}(\tilde{\theta}) \Longrightarrow \theta=\tilde{\theta},
$$

then the model considered in the distribution sense is identifiable with respect to $\theta$.

Proof 3.2. The proof is similar to the proof of Proposition 3.1.

Remark 3.2. It is easy to show that if the $\left(m_{k}\left(y_{\theta}, u\right)\right)_{k=1, \ldots, n}$ are linearly independent then the $\left(T_{m_{k}\left(y_{\theta}, u\right)}\right)_{1 \leq k \leq n}$ are linearly independent too. In the application of section 4, the linear independence assumption of Proposition 3.2 is substituted by the linear independence of the $\left(m_{k}\left(y_{\theta}, u\right)\right)_{k=1, \ldots, n}$.

\subsection{Identifiability result for $\left(\Gamma_{a}\right)$ when $\theta_{a} \in L^{\infty}$}

The solution $\zeta$ of system $\left(\Gamma_{a}\right)$ being piecewise differentiable in time, the output $y_{a}$ is, in general, piecewise differentiable (or just continuous) in time. Using this property, one can obtain a more informative input-output relation taking account the discontinuous points of $y_{a}$ (recall that only derivation with respect to the 
time variable is considered). These new relations can lead to a positive identifiability result.

Let $s$ be the time derivative of highest order of $y_{a}$ appearing in (7). If the function $y_{a}^{(s)}$ can be expressed formally in (7) as a term of the form $d\left(y_{a}, y_{a}^{\prime}, \ldots, y_{a}^{s-1}\right) y_{a}^{(s)}$ where $d$ is a differential polynomial with respect to $y_{a}$ and does not cancel, then (7) can be rewritten as follows:

$$
y_{a}^{(s)}=\tilde{m}_{0}\left(y_{a}\right)+\sum_{k=1}^{n} \gamma_{k}\left(\theta_{a}\right) \tilde{m}_{k}\left(y_{a}\right),
$$

where, for all $k=0, \ldots, n, \tilde{m}_{k}\left(y_{a}\right)$ is a rational function assumed to be locally Lebesgue-integrable in time. Note that we omit the dependence on the input function $u$ to simplify the notation, we will keep this notation in the sequel.

Assume that $y_{a}$ is $s$ times piecewise continuously differentiable in time and let $\left(t_{\nu}\right)_{\nu}$ be the corresponding finite increasing family of discontinuities points. Due to the discontinuities points, the relation (11) should be considered in the distribution sense:

$$
T_{y_{a}^{(s)}}=T_{\tilde{m}_{0}\left(y_{a}\right)}+\sum_{k=1}^{n} \gamma_{k}\left(\theta_{a}\right) T_{\tilde{m}_{k}\left(y_{a}\right)} .
$$

Now, for any $j=0, \ldots, s$, denote the jump at $t_{\nu}$ of $y_{a}^{(j)}$ by

$$
\sigma_{y_{a}, \nu}^{j}\left(\theta_{a}\right):=y_{a}^{(j)}\left(t_{\nu}^{+}\right)-y_{a}^{(j)}\left(t_{\nu}^{-}\right) .
$$

Note that the jump depends upon $\theta_{a}$ since $y_{a}$ itself depends on $\theta_{a}$. Then, jump formula yields

$$
T_{y_{a}}^{(s)}=T_{y_{a}^{(s)}}+\sum_{\nu} \sum_{j=0}^{s-1} \sigma_{y_{a}, \nu}^{j} \delta_{t_{\nu}}^{(s-1-j)},
$$

where $\delta_{t_{\nu}}$ denotes the Dirac distribution at point $t_{\nu}$. Thus, equation (12) can be rewritten

$$
T_{y_{a}}^{(s)}=T_{\tilde{m}_{0}\left(y_{a}\right)}-\sum_{\nu} \sum_{j=0}^{s-1} \sigma_{y_{a}, \nu}^{j}\left(\theta_{a}\right) \delta_{t_{\nu}}^{(s-1-j)}+\sum_{k=1}^{n} \gamma_{k}\left(\theta_{a}\right) T_{\tilde{m}_{k}\left(y_{a}\right)} .
$$




\section{Remark 3.3.}

1. These relations are more informative than (11) since new constraints on the discontinuity points linking the parameters are added to the study of identifiability.

2. This operation can permit to obtain relations well-defined with respect to the solution regularity.

The following proposition gives also a sufficient condition to obtain the identifiability of the parameters.

Proposition 3.3. Assume that the family of distributions $\left(T_{\tilde{m}_{k}\left(y_{a}\right)}\right)_{1 \leq k \leq n}$ is linearly independent. If, for all $\left(\theta_{a}, \tilde{\theta}_{a}\right) \in L^{\infty}\left(\Omega, \mathcal{U}_{p}\right)^{2}$, we have

$$
\left\{\begin{array}{l}
\gamma_{k}\left(\theta_{a}\right)=\gamma_{k}\left(\tilde{\theta}_{a}\right) \\
\forall \nu, \quad \sum_{j=0}^{s-1} \sigma_{y_{a}, \nu}^{j}\left(\theta_{a}\right)=\sum_{j=0}^{s-1} \sigma_{y_{a}, \nu}^{j}\left(\tilde{\theta}_{a}\right) \Longrightarrow \theta_{a}=\tilde{\theta}_{a}
\end{array}\right.
$$

then the model is identifiable with respect to $\theta_{a}$.

Proof 3.3. The proof is similar to the proof of Proposition 3.1 and follows from the linear independence of the families $\left(\delta_{t_{\nu}}^{(j)}\right)_{j=0, \ldots, s}$ and $\left(\tilde{T}_{m_{k}}\left(y_{\theta}\right)\right)_{k=1, \ldots, n}$.

All these input-output polynomials (7), (10), (13) can be used to obtain a first estimate of the identifiable parameters. This will be illustrated at section 5 .

Example 3.2. Consider again Example 3.1 but now assume that $u$ is a piecewise constant function given by

$$
u(t):= \begin{cases}u_{1} & \text { if } t \in\left(0, t_{1}\right) \\ u_{2} & \text { if } t \in\left(t_{1}, T\right)\end{cases}
$$

where $u_{1}, u_{2} \in \mathbb{R}, u_{1} \neq u_{2}$. Then, equation (8) written in distribution sense yields

$$
T_{y}^{\prime \prime}=T_{u}+k\left(u_{2}-u_{1}\right) \delta_{t_{1}}
$$

So, in this way, we obtained a new relation that could permit to identifiate $k$. 


\subsection{Identifiability result on $\left(\Gamma_{b}\right)$ when $\theta_{b}$ is a constant parameter vector}

In this part, we focus on the identifiability of system $\left(\Gamma_{b}\right)$. We are interested in the identifiability of the parameter $\theta_{b}:=\left(\theta_{c}, \theta_{d}\right)$ where $\theta_{c} \in \mathbb{R}^{q_{0}}$ and $\theta_{d}:=\left(d_{i}\right)_{i \in I}$ with $I \subset\left\{q_{0}+1, \ldots, q\right\}$. Let $U:=\left(U_{i}\right)_{i=1, \ldots, q}$ be the solution of $\left(\Gamma_{b}\right)$ and $y_{b}$ be given by (6).

Lemma 3.1. Let $y_{b}$ be given by $y_{b}:=\left(y_{c}, y_{d}\right)$ where

$$
y_{d}:=\left(U_{i}\right)_{i \in I} .
$$

Assume there exists $\nu \in \mathbb{N}$ such that, for all $t \in\left(t_{\nu}, t_{\nu+1}\right)$,

1. there exists a family of positive reals $\left(\lambda_{i}\right)_{i \in I}$ such that

$$
\sum_{i \in I} \lambda_{i}\left(g_{i}\left(\zeta(t), U(t), \theta_{d}\right)-\partial_{t} U_{i}\right)
$$

is independent of $\theta_{d}$,

2. the family $\left(U_{i}(t, \cdot)\right)_{i \in I}$ is linearly independent up to an additive constant in $\Omega$,

3. the $b_{i}$ defined in $B_{i}=d_{i} A+b_{i}$ are supposed to be known.

Then, $\left(\Gamma_{b}\right)$ is identifiable with respect to $\theta_{d}$.

Remark 3.4. In other words, Lemma 3.1 gives a sufficient condition for the identifiability of diffusion coefficients $\left(d_{i}\right)_{i \in I}$ when the associated solutions $\left(U_{i}\right)_{i \in I}$ are known.

Proof 3.4. Let $\theta_{d}, \tilde{\theta}_{d}$ be such that

$$
\forall(t, x) \in\left(t_{\nu}, t_{\nu+1}\right) \times \Omega, \quad y_{b}\left(t, x, \theta_{d}\right)=y_{b}\left(t, x, \tilde{\theta}_{d}\right) .
$$

Then, using assumption (1), we have

$$
\begin{aligned}
\sum_{i \in I} \lambda_{i} B_{i}\left(\theta_{d}\right) U_{i} & =\sum_{i \in I} \lambda_{i}\left(g_{i}\left(\zeta(t), U(t), \theta_{d}\right)-\partial_{t} U_{i}\right) \\
& =\sum_{i \in I} \lambda_{i}\left(g_{i}\left(\zeta(t), U(t), \tilde{\theta}_{d}\right)-\partial_{t} U_{i}\right) \\
& =\sum_{i \in I} \lambda_{i} B_{i}\left(\tilde{\theta}_{d}\right) U_{i}
\end{aligned}
$$


which leads to

$$
\sum_{i \in I} \lambda_{i}\left(d_{i}-\tilde{d}_{i}\right) A U_{i}=0
$$

Thus, $V:=\sum_{i \in I} \lambda_{i}\left(d_{i}-\tilde{d}_{i}\right) U_{i}$ is an eigenvector of $A$ associated with zero eigenvalue. Thus, $V$ is constant. Since, for all $t \in\left(t_{\nu}, t_{\nu+1}\right),\left(U_{i}(t, \cdot)\right)_{i \in I}$ is linearly independent up to an additive constant in $\Omega$, we deduce that for all $i \in I$

$$
\lambda_{i}\left(d_{i}-\tilde{d}_{i}\right)=0
$$

which leads to the result since $\lambda_{i} \neq 0$.

It remains to obtain the identifiability with respect to $\theta_{c}$. To this end, Propositions 3.1 and 3.2 can be used. However, in order to test the linear independence of the family $\left(m_{k}\left(y_{b}\right)\right)_{k=1, \ldots, n}$, the calculus of the wronskian can not be possible due to the lack of regularity of the solutions. In the following lemma, we propose to substitute this test by another one.

Lemma 3.2. Let $\tilde{P}:=\langle P\rangle$. Assume that

1. $\left(\Gamma_{b}\right)$ is identifiable with respect to $\theta_{d}$,

2. the polynomial $P$ defined in (7) with $\theta:=\theta_{c}$ satisfy, for all $k=1, \ldots, n$,

$$
\gamma_{k}\left(\theta_{c}\right)=\gamma_{k}\left(\tilde{\theta}_{c}\right) \Longrightarrow \theta_{c}=\tilde{\theta}_{c}
$$

3. the family of functions $\left(<m_{k}\left(y_{b}\right)>\right)_{k=1, \ldots, n}$ are linearly independent.

Then $\left(\Gamma_{b}\right)$ is identifiable with respect to $\theta_{c}$.

Proof 3.5. The relation $\tilde{P}\left(y_{b}, \theta_{c}\right)-\tilde{P}\left(y_{b}, \tilde{\theta}_{c}\right)$ gives:

$$
\left.\sum_{k=1}^{n}\left(\gamma_{k}\left(\theta_{c}\right)\right)-\gamma_{k}\left(\tilde{\theta}_{c}\right)\right)\left\langle m_{k}\left(y_{b}\right)\right\rangle=0 .
$$

A linear system of solutions in the block of parameters $\gamma_{k}\left(\theta_{c}\right)-\gamma_{k}\left(\tilde{\theta}_{c}\right)$ is deduced. According to Assumption 3, we obtain that $\gamma_{k}\left(\theta_{c}\right)=\gamma_{k}\left(\tilde{\theta}_{c}\right)$ and according to Assumption 2, $\left(\Gamma_{b}\right)$ is identifiable with respect to $\theta_{c}$.

Remark 3.5. 1. If the relation $\tilde{P}$ obtained from (7) by taken its mean value is not well-defined with respect to the time variable, it can be considered in the distribution sense. In that case, for Assumption 3, it is sufficient to prove the linear independence of the family $\left(\left\langle m_{k}\left(y_{b}\right)\right\rangle\right)_{k=1, \ldots, n}$. Indeed, if the family $\left(\left\langle m_{k}\left(y_{b}\right)\right\rangle\right)_{k=1, \ldots, n}$ is linearly independent, then the family $\left(T_{\left\langle m_{k}\left(y_{b}\right)\right\rangle}\right)_{k=1, \ldots, n}$ is also linearly independent. 
2. Another way to deal with the linear independence assumption is to suppose that there exist $n$ distinct points $t_{1}, \ldots, t_{n}$ such that the determinant

$$
\left|\begin{array}{ccc}
\left\langle m_{1}\left(y_{b}\right)\right\rangle\left(t_{1}\right) & \ldots & \left\langle m_{n}\left(y_{b}\right)\right\rangle\left(t_{1}\right) \\
\left\langle m_{1}\left(y_{b}\right)\right\rangle\left(t_{2}\right) & \ldots & \left\langle m_{n}\left(y_{b}\right)\right\rangle\left(t_{2}\right) \\
\vdots & & \vdots \\
\left\langle m_{1}\left(y_{b}\right)\right\rangle\left(t_{n}\right) & \ldots & \left\langle m_{n}\left(y_{b}\right)\right\rangle\left(t_{n}\right)
\end{array}\right|
$$

is not zero.

Indeed, from relation (14), the following linear system in the block of parameters composed of $n$ equations is obtained:

$$
\left.\sum_{k=1}^{n}\left(\gamma_{k}\left(\theta_{c}\right)\right)-\gamma_{k}\left(\tilde{\theta}_{c}\right)\right)\left\langle m_{k}\left(y_{b}\right)\right\rangle\left(t_{j}\right)=0, \quad \text { for } j=1, \ldots, n .
$$

Thus, since the determinant (15) is not zero, following the same argumentation, the identifiability of $\left(\Gamma_{b}\right)$ at $\theta_{c}$ is deduced.

In the numerical applications, the $n$ distinct points $t_{1}, \ldots, t_{n}$ can be chosen randomly to evaluate this determinant.

\section{Application to an epidemiological model}

The identifiability method presented at the previous section is applied on a coupling model describing the transmission and the propagation of the chikungunya disease by Aedes mosquitoes to the human population (See. [20]).

\subsection{Chikungunya transmission model}

The model of [20] couples two sub-systems: the mosquito dynamics model and the transmission virus model between mosquitoes and humans populations. The first one describes the biological mosquito life cycle so that three stages are concerned: $E_{m}$ the number of eggs, $L_{m}$ the number of larvae/pupae and $A_{m}$ the number of adult females. This model is the following

$$
\left\{\begin{array}{l}
E_{m}^{\prime}(t)=b A_{m}(t)\left(1-\frac{E_{m}(t)}{K_{E}}\right)-(s+d) E_{m}(t) \\
L_{m}^{\prime}(t)=s E_{m}(t)\left(1-\frac{L_{m}(t)}{K_{L}}\right)-\left(s_{L}+d_{L}\right) L_{m}(t) \\
A_{m}^{\prime}(t)=s_{L} L_{m}(t)-d_{m} A_{m}(t)
\end{array}\right.
$$


where $K_{E}>0$ (resp. $K_{L}=K_{E} / 2$ ) is the carrying capacity of $E_{m}$ (resp. carrying capacity of $L_{m}$ ), $s_{L}>0$ (resp. $s>0$ ) is transfer rate from $L_{m}$ to $A_{m}$ (resp. from $E_{m}$ to $\left.L_{m}\right), b>0$ is the intrinsic rate, $d>0, d_{L}>0$ and $d_{m}>0$ are the mortality rate for $E_{m}, L_{m}$ and $A_{m}$ respectively.

To construct the second model, that is the transmission virus one, the human and mosquito populations had been first considered in [20] and divided in subpopulations before doing a change of variables to obtain densities. In this paper, only the final version concerning densities is studied. In this model, three stages are considered: $I_{m}$ the density of infective mosquitoes; $S_{H}$ the density of susceptible humans; $I_{H}$ the density of infected humans. The transmission virus model is

$$
\left\{\begin{array}{l}
I_{m}^{\prime}(t)=-\left(s_{L} \frac{L_{m}(t)}{A_{m}(t)}+\beta_{m} I_{H}(t)\right) I_{m}(t)+\beta_{m} I_{H}(t) \\
S_{H}^{\prime}(t)=-\left(b_{H}+\beta_{H} I_{m}(t)\right) S_{H}(t)+b_{H} \\
I_{H}^{\prime}(t)=\beta_{H} I_{m}(t) S_{H}(t)-\left(\gamma+b_{H}\right) I_{H}(t)
\end{array}\right.
$$

where $\beta_{H}>0$ (resp. $\beta_{m}>0$ ) is the infectious contact rate between susceptible humans and vectors (resp. susceptible mosquitoes and infected humans), $\gamma>0$ is the human recovery rate, $b_{H}>0$ is the human birth rate.

Under the same conditions as in [21], a continuous model in space is proposed in this paper. Contrary to the displacement of mosquitoes limited to a few meters, human mobility is considered using diffusion terms (See e.g. [22]). Hence, Model (17) is rewritten as follows:

$$
\left\{\begin{array}{l}
\left\{\begin{array}{l}
\partial_{t} E_{m}(t, x)=b A_{m}(t, x)\left(1-\frac{E_{m}(t, x)}{K_{E}(t, x)}\right)-(s+d) E_{m}(t, x), \\
\partial_{t} L_{m}(t, x)=s E_{m}(t, x)\left(1-\frac{L_{m}(t, x)}{K_{L}(t, x)}\right)-\left(s_{L}+d_{L}\right) L_{m}(t, x), \\
\partial_{t} A_{m}(t, x)=s_{L} L_{m}(t, x)-d_{m} A_{m}(t, x),
\end{array}\right. \\
\left\{\begin{array}{l}
\partial_{t} I_{m}(t, x)=-\left(s_{L} \frac{L_{m}(t, x)}{A_{m}(t, x)}+\beta_{m} I_{H}(t, x)\right) I_{m}(t, x)+\beta_{m} I_{H}(t, x), \\
\partial_{t} S_{H}(t, x)=-\left(b_{H}+\beta_{H} I_{m}(t, x)\right) S_{H}(t, x)+b_{H}+d_{1} \Delta S_{H}(t, x), \\
\partial_{t} I_{H}(t, x)=\beta_{H} I_{m}(t, x) S_{H}(t, x)-\left(\gamma+b_{H}\right) I_{H}(t, x)+d_{2} \Delta I_{H}(t, x),
\end{array}\right.
\end{array}\right.
$$

where $d_{1}, d_{2}>0$ are diffusion coefficients, $x$ is in a bounded domain $\Omega \subset \mathbb{R}^{n}$ with $C^{2}$-boundary and $t>0$. The model is completed with the following initial 
conditions

$$
\begin{aligned}
\left(E_{m}(0, x), L_{m}(0, x), A_{m}(0, x)\right) & =\left(E_{0}(x), L_{0}(x), A_{0}(x)\right), \\
\left(I_{m}(0, x), S_{H}(0, x), I_{H}(0, x)\right) & =\left(I_{0}^{m}(x), S_{0}^{H}(x), I_{0}^{H}(x)\right) .
\end{aligned}
$$

Furthermore, we assume that there there is no population flux across the boundary $\partial \Omega$ of $\Omega$, thus the model is completed by homogeneous Neumann boundary conditions:

$$
\frac{\partial S_{H}}{\partial \nu}=\frac{\partial I_{H}}{\partial \nu}=0 \text { in } \mathbb{R}_{+} \times \partial \Omega
$$

where $\nu$ is the unit outward normal at $\partial \Omega$.

An identifiability study of this model was proposed in [37] but with constant carrying capacities and continuous initial conditions and consequently in the framework of continuous solutions. In this paper, we generalize the results of [37] to the $L^{2}$ framework. More precisely, we make the following assumptions:

1. $\left(E_{0}, L_{0}, A_{0}\right) \in C(\bar{\Omega})^{3}, I_{0}^{m} \in C(\Omega)$ and $\left(S_{0}^{H}, I_{0}^{H}\right) \in L^{2}(\Omega)^{2}$.

2. The carrying capacities of mosquitoes stages $K_{E}$ and $K_{L}$ satisfy

$$
K_{E}, K_{L} \in L^{\infty}([0, \infty) \times \Omega) \quad \text { with } \quad K_{L}(t, x)=K_{E}(t, x) / 2 \neq 0, \quad \text { a.e. }(t, x) \in \mathbb{R}_{+} \times \Omega \text {. }
$$

3. Moreover, $K_{E}$ and $K_{L}$ are piecewise constant functions in time that can correspond to a brutal change in the environment.

\subsection{Existence and uniqueness of the solutions}

\subsubsection{The ODE system}

We set

$$
\Sigma:=\left\{(E, L, A) \in C(\bar{\Omega})^{3} \mid 0 \leq E \leq k, 0 \leq L \leq \frac{k}{2}, 0 \leq A \leq \frac{s_{L} k}{2 d_{m}}\right\},
$$

where, to simplify notations, we set $k:=\left\|K_{E}\right\|_{\infty}>0$.

A simple adaptation of Lemmas 4.2 and 4.3 of [20], from the case $K_{E}$ constant to the case $K_{E}$ depending of $x$ and piecewise constant function in time, leads to the following result:

Proposition 4.1. Let $\left(E_{0}, L_{0}, A_{0}\right) \in \Sigma$. We set

$$
R:=\frac{s_{L}}{d_{m}} \frac{b}{s+d} \frac{s}{s_{L}+d_{L}} .
$$


Then, if $R>1$, system (18)-(a) admits a unique solution $\left(E_{m}, L_{m}, A_{m}\right)$ in the space of differentiable piecewise functions $C_{l}^{1}([0, \infty) ; \operatorname{int}(\Sigma))^{3}$ where int $(\Sigma)$ is the interior of $\Sigma$.

\section{Remark 4.1.}

1. Note that the discontinuous points of $E_{m}, L_{m}$ and $A_{m}$ are the same and are those of $K_{E}$.

2. From [20], the condition of survival of eggs, larvae and adults populations of mosquitoes is given by $R>1$. Afterwards, we suppose that $R>1$ so that $E_{m}, L_{m}, A_{m}>0$. In particular, we make the following assumption:

$(\mathcal{H}) R>1$ and there exists $c>0$ such that, for any $t \geq 0$,

$$
\zeta_{3}(t):=A_{m}(t, \cdot)>c \text {. }
$$

\subsubsection{The PDE system}

Since $(\mathcal{H})$ is satisfied, we have

$$
r:=\frac{L_{m}}{A_{m}} \in C_{l}^{1}([0, \infty) ; C(\bar{\Omega})) .
$$

By setting $U:=\left(I_{m}, S_{H}, I_{H}\right)$, system (18)-(b) is of the form (2) where the Banach space $X$ is given by

$$
X:=\left\{\left(u_{1}, u_{2}, u_{3}\right) \mid u_{1} \in C(\bar{\Omega}), u_{2}, u_{3} \in L^{2}(\Omega)\right\},
$$

and the operator $B$ is defined by

$$
B:=\operatorname{diag}\left(I,-d_{1} A+b_{H},-d_{2} A+\gamma+b_{H}\right),
$$

where $A$ is the realization of the Laplacian in $L^{2}(\Omega)$ with Neuman homogeneous boundary condition on $\partial \Omega$, and $I$ is the identity operator of $C(\bar{\Omega})$. Furthermore, the nonlinear operator $G(t, \cdot)$ is given by

$$
G(t, U):=\left(-\left(s_{L} r(t)+\beta_{m} u_{3}-1\right) u_{1}+\beta_{m} u_{3},-\beta_{H} u_{1} u_{3}+b_{H}, \beta_{H} u_{1} u_{2}\right) .
$$

We prove in the appendix, section 7, that $G$ satisfies (3) and thus (18)-(b) admits a unique local solution

$$
U \in C_{l}\left(\left[0, T_{U_{0}}\right] ; D(B)\right) \cap C_{l}^{1}\left(\left(0, T_{U_{0}}\right] ; X\right)
$$

where $U_{0}:=\left(I_{0}^{m}, S_{0}^{H}, I_{H}^{0}\right)$.

Moreover, setting

$$
X_{+}:=\left\{\left(u_{1}, u_{2}, u_{3}\right) \in X \mid u_{1}, u_{2}, u_{3} \geq 0\right\},
$$

we have the following theorem which states that the solution is global. 
Theorem 4.1. Under the assumption $(\mathcal{H})$, for any $\left(I_{0}^{m}, S_{0}^{H}, I_{0}^{H}\right) \in X_{+}$, (18)-(b) possesses a unique non-negative global solution such that

$$
\left\{\begin{array}{l}
I_{m} \in C_{l}^{1}([0, \infty) ; C(\bar{\Omega})) \\
S_{H}, I_{H} \in C_{l}\left([0, \infty) ; H_{N}^{2}(\Omega)\right) \cap C_{l}^{1}\left((0, \infty) ; L^{2}(\Omega)\right) .
\end{array}\right.
$$

The proof of the theorem is given in the appendix, section 7.

\subsection{Identifiability result}

The parameters whose values are not directly accessible from the field are $s$, $s_{L}$ for the system (18)-(a) and $\beta_{H}, \beta_{m}, d_{1}, d_{2}$ for the system (18)-(b).

In the case of the ODEs model (16)-(17), an identifiable study had been done in [27] and a procedure based on input-output polynomials had been proposed in [32]. In [37], the identifiability model (18) was studied but in the case of very regular functions. Namely, for system (18)-(a), the solutions were $C^{\infty}$ and for system (18)-(b) the solutions were continuous. We consider the same assumptions on the measured outputs, that is $L_{m}, S_{H}, I_{H}$ can be measured.

Indeed, some weekly informations on the number of mosquitoes larvae can be obtained owing to the use of water tanks in which the mosquitoes female lay their eggs. Then, institutes for Health Care can collect periodically data on the number of new infections. Thus, we assume that the number of susceptible humans $S_{H}$, infected humans $I_{H}$ and larvae $L_{m}$ can be considered as measured variables in $\Omega$ during a finite time interval.

The main result of the paper is the following:

Theorem 4.2. Assume the constants $b, d, d_{L}, d_{m}, b_{H}, \gamma$ are known and there exists $\nu \in \mathbb{N}$ such that

- for all $(t, x) \in\left(t_{\nu}, t_{\nu+1}\right) \times \Omega, L_{m}(t, x), S_{H}(t, x)$ and $I_{H}(t, x)$ are the outputs of the model,

- for any $t \in\left(t_{\nu}, t_{\nu+1}\right), S_{H}(t, \cdot)$ and $I_{H}(t, \cdot)$ are linearly independent up to an additive constant in $\Omega$.

We recall that the notation $<., .>$ is the mean on all the space $\Omega$. Then, system (18) is identifiable with respect to $s, s_{L}, A_{0}, d_{1}$ and $d_{2}$.

Let $\tilde{I}_{m}:=\beta_{H} I_{m}$. Under the same assumptions, if there exists $\tilde{t} \in\left(t_{\nu}, t_{\nu+1}\right)$

such that $\left\langle I_{H} S_{H}^{2}\right\rangle(\tilde{t}) \neq 0$ and $\left\langle\tilde{I}_{m} I_{H} S_{H}^{2}\right\rangle(\tilde{t}) \neq 0$, then system (18) is identifiable with respect to $\beta_{m}$ and $\beta_{H}$. 


\section{Remark 4.2.}

1. According to equation (18)-(b), $\tilde{I}_{m}$ can be expressed almost everywhere only in function of the measured variable $S_{H}\left(\right.$ or $\left.I_{H}\right)$ and of the identifiable parameter $d_{1}\left(\right.$ or $\left.d_{2}\right)$. Consequently, $\tilde{I}_{m}$ is considered as a measured variable almost everywhere.

2. Note that a supplementary result is obtained on the identifiability of the initial condition $A_{0} \in C(\bar{\Omega})$. Therefore, by an optimization procedure based on the identifiability study, the values of $A_{0}$ and the other unknown parameters can be obtained in a unique way from the knowledge of $L_{m}, S_{H}$ and $I_{H}$.

3. An example of the estimation of $d_{1}$ and $d_{2}$ is given at section 5 .

\subsection{Proof of Theorem 4.2}

We proceed in two steps. In the first step, we show that (18)-(a) is identifiable with respect to $\theta_{a}:=\left(s, s_{L}, A_{0}\right)$. In the second step, we show that (18)-(b) is identifiable with respect to $\theta_{b}:=\left(\beta_{m}, \beta_{H}, d_{1}, d_{2}\right)$.

First step. For $x$ fixed in $\Omega$, let us introduce the following notations:

$$
\forall t \in[0, \infty), \quad \zeta_{1}(t):=\frac{E_{m}(t, x)}{K_{E}(t, x)}, \quad \zeta_{2}(t):=\frac{L_{m}(t, x)}{K_{E}(t, x)}, \quad \zeta_{3}(t):=\frac{A_{m}(t, x)}{K_{E}(t, x)} .
$$

In all the sequel, we denote by $\left(t_{\nu}\right)_{\nu}$ the finite increasing family of discontinuity time points of $K_{E}$ (and so of $\zeta_{1}, \zeta_{2}$ and $\zeta_{3}$ ). First, to obtain simpler polynomials, the third equation of system (18)-(a) is integrated before using the package DifferentialAlgebra of Maple.

Integration of the third equation of system (18)-(a) on any interval $\left(t_{\nu}, t_{\nu+1}\right)$ yields

$$
\forall t \in\left(t_{\nu}, t_{\nu+1}\right), \quad \zeta_{3}(t)=\zeta_{3}(0) e^{-d_{m} t}+s_{L} \int_{t_{\nu}}^{t_{\nu+1}} \zeta_{2}(s, x) e^{-d_{m}(t-s)} d s .
$$

Then, we set

$\forall t \in\left(t_{\nu}, t_{\nu+1}\right), \quad \tilde{\zeta}_{2, \nu}(t, x):=\int_{t_{\nu}}^{t_{\nu+1}} \zeta_{2}(s, x) e^{-d_{m}(t-s)} d s \quad$ and $\quad \lambda(t):=e^{-d_{m} t}$.

To simplify the notations, we let $\tilde{\zeta}_{2, \nu}=\tilde{\zeta}_{2}$. Since $\tilde{\zeta}_{2}$ depends only on $d_{m}$ and the measured variable $\zeta_{2}$, it can be also considered as a measured variable. Thus, we have three observations

$$
y_{a, 1}:=\zeta_{2}, \quad y_{a, 2}:=\tilde{\zeta}_{2} \quad \text { and } \quad y_{a, 3}:=\lambda
$$


Then, system (18)-(a) can be rewritten:

$$
\left\{\begin{aligned}
\partial_{t} \zeta_{1} & =b \zeta_{3}\left(1-\zeta_{1}\right)-(s+d) \zeta_{1} \\
\partial_{t} \zeta_{2} & =s \zeta_{1}\left(1-2 \zeta_{2}\right)-\left(s_{L}+d_{L}\right) \zeta_{2} \\
\zeta_{3} & =\zeta_{3}(0) \lambda+s_{L} \tilde{\zeta}_{2} \\
\partial_{t} \tilde{\zeta}_{2} & =\zeta_{2}-d_{m} \tilde{\zeta}_{2} \\
\partial_{t} \lambda & =-d_{m} \lambda \\
y_{a, 1} & =\zeta_{2}, \quad y_{a, 2}=\tilde{\zeta}_{2}, \quad y_{a, 3}=\lambda
\end{aligned}\right.
$$

with the initial conditions:

$$
\left(\zeta_{1}(0, x), \zeta_{2}(0, x), \zeta_{3}(0, x), \tilde{\zeta}_{2}(0, x), \lambda(0)\right)=\left(\zeta_{1,0}(x), \zeta_{2,0}(x), \zeta_{3,0}(x), 0,1\right) .
$$

The Rosenfeld-Groebner algorithm ${ }^{1}$ is then applied with (20) and the elimination order $^{2}$

$$
\left[\zeta_{3,0}, s, s_{L}\right] \prec\left[y_{a, 1}, y_{a, 2}, y_{a, 3}\right] \prec\left[\zeta_{1}, \zeta_{2}, \zeta_{3}, \tilde{\zeta}_{2}, \lambda\right]
$$

The software gives the characteristic presentation composed of the following polynomials:

$Q_{1}=-\zeta_{3,0} y_{a, 3}-s_{L} y_{a, 2}+\zeta_{3}, \quad Q_{2}=\zeta_{1}\left(2 y_{a, 1}-1\right) s+y_{a, 1} s_{L}+d_{L} y_{a, 1}+\partial_{t} y_{a, 1}$

and

$$
\begin{aligned}
& P=-2 \partial_{t}\left(y_{a, 1}^{2}\right)-\partial_{t}^{2} y_{a, 1}+2 y_{a, 1} \partial_{t}^{2} y_{a, 1}+b s \zeta_{3,0} y_{a, 3}+\left(d d_{L}+d s_{L}+d_{L} s\right. \\
& \left.+s s_{L}\right)\left(2 y_{a, 1}^{2}-y_{a, 1}\right)+\left(2 b d_{L} \zeta_{3,0}+4 b s \zeta_{3,0}+2 b s_{L} \zeta_{3,0}\right) y_{a, 1}^{2} y_{a, 3} \\
& +\left(-b d_{L} \zeta_{3,0}-4 b s \zeta_{3,0}-b s_{L} \zeta_{3,0}\right) y_{a, 1} y_{a, 3}+b s s_{L} y_{a, 2} \\
& +\left(-b d_{L} s_{L}-4 b s s_{L}-b s_{L}^{2}\right) y_{a, 1} y_{a, 2}+\left(2 b d_{L} s_{L}+4 b s s_{L}+2 b s_{L}^{2}\right) y_{a, 1}^{2} y_{a, 2} \\
& +b \zeta_{3,0}\left(-y_{a, 3} \partial_{t} y_{a, 1}+2 y_{a, 1} y_{a, 3} \partial_{t} y_{a, 1}\right)+(2 d+2 s) y_{a, 1} \partial_{t} y_{a, 1} \\
& -\left(d+d_{L}+s+s_{L}\right) \partial_{t} y_{a, 1}+b s_{L}\left(2 y_{a, 1} \partial_{t} y_{a, 1} y_{a, 2}-\partial_{t} y_{a, 1} y_{a, 2}\right) .
\end{aligned}
$$

From the two first polynomials $Q_{1}, Q_{2}, \zeta_{1}$ and $\zeta_{3}$ can be expressed according to $y_{a, 1}, y_{a, 2}, y_{a, 3}$. The third one, $P$, links the outputs and the parameters $s, s_{L}$ and

\footnotetext{
${ }^{1}$ This algorithm is implemented in in the package DifferentialAlgebra of Maple.

${ }^{2}$ Note that the equations $\dot{\xi}_{3,0}=0, \dot{s}=0$ and $\dot{s}_{L}=0$ had been added to $\left(\Gamma_{a}\right)$ before the elimination procedure.
} 
$\zeta_{3,0}$ of the model, it is also an input-output polynomial. Since solutions of (18) are in $C_{l}^{1}([0, \infty) ; \mathcal{C})$ and $P$ contains derivatives of second order in time, $P$ is not well-defined with respect to the regularity of the solution. Let us show how to obtain a regular relation.

In considering $P$ in the distribution sense and in changing the term $y_{a, 1} \partial_{t}^{2} y_{a, 1}$ to $\partial_{t}\left(y_{a, 1} \partial_{t} y_{a, 1}\right)-\left(\partial_{t} y_{a, 1}\right)^{2}$ the following polynomial $\tilde{P}$ in the distribution sense is obtained:

$$
\begin{aligned}
& \tilde{P}=-2 T_{\partial_{t}\left(y_{a, 1}^{2}\right)}-T_{y_{a, 1}}^{\prime \prime}+2 T_{y_{a, 1} \partial_{t} y_{a, 1}}^{\prime}-2 T_{\left(\partial_{t} y_{a, 1}\right)^{2}}+\gamma_{1}\left(\theta_{a}\right) T_{y_{a, 3}}+\gamma_{2}\left(\theta_{a}\right) T_{2 y_{a, 1}^{2}-y_{a, 1}} \\
& +\gamma_{2}\left(\theta_{a}\right) T_{y_{a, 1}}+\gamma_{3}\left(\theta_{a}\right) T_{y_{a, 1}^{2} y_{a, 3}}+\gamma_{4}\left(\theta_{a}\right) T_{y_{a, 1} y_{a, 3}}+\gamma_{5}\left(\theta_{a}\right) T_{y_{a, 2}}+\gamma_{6}\left(\theta_{a}\right) T_{y_{a, 1} y_{a, 2}} \\
& +\gamma_{7}\left(\theta_{a}\right) T_{y_{a, 1}^{2} y_{a, 2}}+\gamma_{8}\left(\theta_{a}\right) T_{-y_{a, 3} \partial_{t} y_{a, 1}+2 y_{a, 1} y_{a, 3} \partial_{t} y_{a, 1}} \\
& +\gamma_{9}\left(\theta_{a}\right) T_{y_{a, 1} \partial_{t} y_{a, 1}}-\gamma_{10}\left(\theta_{a}\right) T_{y_{a, 1}}^{\prime}+\gamma_{11}\left(\theta_{a}\right) T_{2 y_{a, 1} \partial_{t} y_{a, 1} y_{a, 2}-\partial_{t} y_{a, 1} y_{a, 2}}
\end{aligned}
$$

where

$$
\begin{aligned}
\gamma\left(\theta_{a}\right):= & \left(\gamma_{k}\left(\theta_{a}\right)\right)_{1 \leq k \leq 11} \\
:= & \left(b s \zeta_{3,0}, d d_{L}+d s_{L}+d_{L} s+s s_{L}\right. \\
& 2 b d_{L} \zeta_{3,0}+4 b s \zeta_{3,0}+2 b s_{L} \zeta_{3,0},-b d_{L} \zeta_{3,0} \\
& -4 b s \zeta_{3,0}-b s_{L} \zeta_{3,0}, b s s_{L},-b d_{L} s_{L}-4 b s s_{L}-b s_{L}^{2} \\
& 2 b d_{L} s_{L}+4 b s s_{L}+2 b s_{L}^{2}, b \zeta_{3,0}, 2 d+2 s \\
& \left.-\left(d+d_{L}+s+s_{L}\right), b s_{L}\right) .
\end{aligned}
$$

Remark 4.3. From the observation $L_{m}$ and by using $\tilde{P}$, the unknown parameters $s, s_{L}$ and $A_{0}(x)$ can be estimated for all $x \in \Omega$.

Let us prove that the model is identifiable with Proposition 3.2. Using Maple, we obtain that the functional determinant of the functions $\left\{y_{a, 3}, 2 y_{a, 1}^{2}-y_{a, 1}\right.$, $y_{a, 1}^{2} y_{a, 3}, y_{a, 1} y_{a, 3}, y_{a, 2}, y_{a, 1} y_{a, 2}, y_{a, 1}^{2} y_{a, 2},-y_{a, 3} \dot{y}_{a, 1}+2 y_{a, 1} y_{a, 3} \dot{y}_{a, 1}, y_{a, 1} \dot{y}_{a, 1}, \dot{y}_{a, 1}$, $\left.2 y_{a, 1} \dot{y}_{a, 1} y_{a, 2}-\dot{y}_{a, 1} y_{a, 2}\right\}$ is not identically equal to zero. Then, solving the algebraic system $\gamma\left(\theta_{a}\right)=\gamma\left(\tilde{\theta}_{a}\right)$ with the Groebner basis implemented in maple leads to $\theta_{a}=\tilde{\theta}_{a}$. Consequently, applying Proposition 3.2, the model is identifiable at the parameters $s, s_{L}$, and $\zeta_{3,0}(x)$ for each $x \in \Omega$.

Second step. Since $S_{H}$ and $I_{H}$ are assumed to be known for all $(t, x) \in\left(t_{\nu}, t_{\nu+1}\right) \times$ $\Omega$, the equalities $y_{b, 1}=S_{H}$ and $y_{b, 2}=I_{H}$ are added to system (18)-(b) which is 
rewritten in the following form:

$$
\left\{\begin{array}{l}
\partial_{t} I_{m}=-\left(s_{L} r+\beta_{m} I_{H}\right) I_{m}+\beta_{m} I_{H}, \\
\partial_{t} S_{H}=-\left(b_{H}+\beta_{H} I_{m}\right) S_{H}+b_{H}+d_{1} \Delta S_{H}, \\
\partial_{t} I_{H}=\beta_{H} I_{m} S_{H}-\left(\gamma+b_{H}\right) I_{H}+d_{2} \Delta I_{H}, \\
y_{b, 1}=S_{H}, y_{b, 2}=I_{H} .
\end{array}\right.
$$

where $r:=\frac{\zeta_{2}}{\zeta_{3}}$ is known according to the previous subsection.

The parameters to identify being $\beta_{m}, \beta_{H}, d_{1}, d_{2}$, we denote $\theta_{b}:=\left(\beta_{m}, \beta_{H}, d_{1}, d_{2}\right)$. The initial conditions are in that case $\left(I_{m}(0, \cdot), S_{H}(0, \cdot), I_{H}(0, \cdot)\right)=\left(I_{0}^{m}, S_{0}^{H}, I_{0}^{H}\right)$.

Following section 3.3, the identifiability of the parameters is done in two steps: first the identifiability study of $d_{1}$ and $d_{2}$ by using Lemma 3.1, then the identifiability study of $\beta_{m}$ and $\beta_{H}$ by using Proposition 3.3.

Identifiability of $d_{1}$ and $d_{2}$.

Summing the second equation and the third equation of $\left(\Gamma_{b}\right)$ yields:

$$
\begin{aligned}
d_{1} \Delta y_{b, 1}(t, \cdot)+d_{2} \Delta y_{b, 2}(t, \cdot)= & \partial_{t} y_{b, 1}(t, \cdot)+\partial_{t} y_{b, 2}(t, \cdot)+b_{H}\left(y_{b, 1}(t, \cdot)-1\right) \\
& +\left(\gamma+b_{H}\right) y_{b, 2}(t, \cdot) .
\end{aligned}
$$

The right hand-side of (21) does not depend on $d_{1}$ and $d_{2}$, and the functions $y_{b, 1}$ and $y_{b, 2}$ are supposed to be linearly independent. According to Lemma 3.1, the model is identifiable with respect to $d_{1}$ and $d_{2}$.

Identifiability of $\beta_{m}$ and $\beta_{H}$.

From the package DifferentialAlgebra of Maple, we obtain the following input-output polynomial:

$$
\begin{aligned}
& -\left(-b_{H} s_{L} r-s_{L} r \gamma\right) y_{b, 2} y_{b, 1}-\left(\gamma+b_{H}\right) y_{b, 2} \partial_{t} y_{b, 1}-\left(-s_{L} r-b_{H}-\gamma\right) y_{b, 1} \partial_{t} y_{b, 2} \\
& -d_{2} r s_{L} y_{b, 1} \Delta y_{b, 2}-\left(d_{2} \partial_{t} \Delta y_{b, 2}-\partial_{t t} y_{b, 2}\right) y_{b, 1}-\partial_{t} y_{b, 2} \partial_{t} y_{b, 1}+d_{2} \partial_{t} y_{b, 1} \Delta y_{b, 2} \\
& =\beta_{m}\left(\left(-\gamma-b_{H}\right) y_{b, 2}^{2} y_{b, 1}-y_{b, 2} y_{b, 1} \partial_{t} y_{b, 2}+d_{2} y_{b, 2} y_{b, 1} \Delta y_{b, 2}\right)+\beta_{H} \beta_{m} y_{b, 2} y_{b, 1}^{2}
\end{aligned}
$$

Since the function $y_{b, 1}=S_{H}$ and $y_{b, 2}=I_{H}$ are in $C_{l}^{1}\left((0, \infty), L^{2}(\Omega)\right)$, the previous polynomial is considered in the distribution sense.

We apply Lemma 3.2. Since the model is identifiable with respect to $d_{1}$ and $d_{2}$, Assumption 1 of Lemma 3.2 is satisfied. The exhaustive summary of the inputoutput relation (22) being $\left(\beta_{m}, \beta_{H} \beta_{m}\right)$, Assumption 2 of Lemma 3.2 is easily verified. 
It remains to show Assumption 3. We set

$m_{1}:=\left(-\gamma-b_{H}\right) y_{b, 2}^{2} y_{b, 1}-y_{b, 2} y_{b, 1} \partial_{t} y_{b, 2}+d_{2} y_{b, 2} y_{b, 1} \Delta y_{b, 2} \quad$ and $\quad m_{2}:=y_{b, 2} y_{b, 1}^{2}$.

$m_{1}$ can be rewritten:

$$
m_{1}=y_{b, 2} y_{b, 1}\left(\left(-\gamma-b_{H}\right) y_{b, 2}-\partial_{t} y_{b, 2}+d_{2} \Delta y_{b, 2}\right)=-y_{b, 2} y_{b, 1}^{2} \tilde{I}_{m}
$$

Thus, the Wronksian of $\left(\left\langle m_{1}\right\rangle,\left\langle m_{2}\right\rangle\right)$ is equal to:

$$
\left\langle y_{b, 2} y_{b, 1}^{2}\right\rangle\left\langle\left(\partial_{t} \tilde{I}_{m}\right) y_{1}^{2} y_{2}\right\rangle
$$

By assumption, there exists a time point $\tilde{t} \in\left(t_{\nu}, t_{\nu+1}\right)$ such that this Wronskian does not vanish. Thus, from Lemma 3.2 and Remark 3.5, the model is identifiable with respect to $\beta_{m}$ and $\beta_{H}$.

Remark 4.4. The proof of Proposition 4.2 provides two input-output polynomials (21) and (22). To illustrate a way to use them in a parameter estimation procedure, we propose, in the following section, a method to estimate $d_{1}$ and $d_{2}$ based on (21). The procedure does not require any knowledge of the parameter values. This first estimate of $d_{1}$ and $d_{2}$ can be used by a local algorithm to improve their estimation.

\section{Example of the estimation of the two parameters $d_{1}$ and $d_{2}$}

This part is devoted to a numerical simulation estimating the parameters $d_{1}$ and $d_{2}$ based on (21). We follow the following procedure:

- We solve (18) with known parameters and thus we obtain approximations of $S_{H}$ and $I_{H}$.

- These approximations permit to simulate measured states $S_{H}^{\text {meas }}$ (resp. $I_{H}^{\text {meas }}$ ) as normal distributions around $S_{H}$ (resp. $I_{H}$ ) with variance $\left(\sigma S_{H}\right)^{2}$ (resp. $\left(\sigma I_{H}\right)^{2}$ ), where $\sigma \in \mathbb{R}_{+}$. The constant $\sigma$ is chosen in order to obtain the desired error between $S_{H}$ and $S_{H}^{\text {meas }}$, and also between $I_{H}$ and $I_{H}^{\text {meas }}$.

- We apply the parameter estimation procedure with the measured states $S_{H}^{\text {meas }}$ and $I_{H}^{m e a s}$ to recover the parameters $d_{1}$ and $d_{2}$.

Let us first present the simulations data. 


\begin{tabular}{l|l|l} 
Parameters & Description & Value \\
\hline$b$ & Oviposition rate & 6 \\
$K_{E}$ & Egg carrying capacity & 1000 \\
$s$ & Egg transformation rate & 0.7 \\
$s_{L}$ & Larva transformation rate & 0.5 \\
$d$ & Egg mortality rate & 0.2 \\
$d_{L}$ & Larva mortality rate & 0.2 \\
$d_{m}$ & Female adult mortality rate & 0.25 \\
$b_{H}$ & Human birth/death rate & 0.0000457 \\
$\beta_{H}$ & Infection rate: vector to human & 0.75 \\
$\beta_{m}$ & Infection rate: human to vector & 0.5 \\
$\gamma$ & Human recovery rate & 0.1428 \\
\hline
\end{tabular}

Table 1: Parameters values

\subsection{Simulations}

We use the parameters values from Table 1 of [19] (see also [21]) and recalled in Table 1. Moreover, we set $d_{1}=0.1$ and $d_{2}=0.01$ so that susceptible populations diffuse through the domain faster than the infected populations. The domain considered is $\Omega:=(0,1)^{2}$ and the final time is $T=20$.

At the initial time, the variables $\zeta_{i}$ are taken constant while the infected humans are supposed to be concentrated in the center of the domain, which can correspond for example to an hospital, and the susceptible humans are located at the extremities of the domain. More precisely, the initial conditions are given by:

$$
\left\{\begin{array}{l}
\zeta_{1}(t=0, x, y)=0.1, \zeta_{2}(t=0, x, y)=0.04, \zeta_{3}(t=0, x, y)=0.01 \\
I_{m}(t=0, x, y)=0.2 \\
S_{H}(t=0, x, y)=1-z(x, y) \\
I_{H}(t=0, x, y)=z(x, y)
\end{array}\right.
$$

where

$$
z(x, y):= \begin{cases}e^{-\frac{1}{1} \frac{1}{4}-\left(x-\frac{1}{2}\right)^{2}-\left(y-\frac{1}{2}\right)^{2}}, & \text { if }\left(x-\frac{1}{2}\right)^{2}+\left(y-\frac{1}{2}\right)^{2}<\frac{1}{4} \\ 0 & \text { else. }\end{cases}
$$

Then, we compute approximations of $S_{H}$ and $I_{H}$ by solving the system (18), with the exact parameter values, by an explicit finite difference method. 
The measured states $S_{H}^{\text {meas }}$ and $I_{H}^{\text {meas }}$ are constructed by using the function randn of Matlab, where the coefficient $\sigma$ is chosen in order to have a maximal value of the relative error between $S_{H}$ and $S_{H}^{\text {meas }}$ (and between $I_{H}$ and $I_{H}^{\text {meas }}$ ) equal to $1 \%, 5 \%, 10 \%$ and $20 \%$.

\subsection{The numerical input-output method for estimating $d_{1}$ and $d_{2}$}

The relation (21) is given in distribution sense by:

$$
\begin{aligned}
& d_{1} \int_{\Omega} S_{H}(t, x) \Delta \psi(x) d x+d_{2} \int_{\Omega} I_{H}(t, x) \Delta \psi(x) d x \\
& =\int_{\Omega}\left(\partial_{t} S_{H}(t, x)+\partial_{t} I_{H}(t, x)\right) \psi(x) d x \\
& +\left(\gamma+b_{H}\right) \int_{\Omega} I_{H}(t, x) \psi(x) d x-b_{H} \int_{\Omega}\left(1-S_{H}(t, x)\right) \psi(x) d x,
\end{aligned}
$$

for all $\psi \in C_{c}(\Omega)$. Integrating this relation on $(0, t)$, we obtain

$$
\begin{aligned}
& d_{1} \int_{0}^{t}\left(\int_{\Omega} S_{H}(\tau, s) \Delta \psi(s) d s\right) d \tau+d_{2} \int_{0}^{t}\left(\int_{\Omega} I_{H}(\tau, s) \Delta \psi(s) d s\right) d \tau \\
& =\int_{\Omega}\left(S_{H}(t, s)+I_{H}(t, s)\right) \psi(s) d s-\int_{\Omega}^{t}\left(S_{H}(0, s)+I_{H}(0, s)\right) \psi(s) d s \\
& +\left(\gamma+b_{H}\right) \int_{0}^{t}\left(\int_{\Omega} I_{H}(\tau, s) \psi(s) d s\right) d \tau \\
& -b_{H} \int_{0}^{t}\left(\int_{\Omega}\left(1-S_{H}(\tau, s)\right) \psi(s) d s\right) d \tau
\end{aligned}
$$

for all $\psi \in C_{c}(\Omega)$ and all $t>0$.

We consider the following test function:

$$
\psi(x, y):= \begin{cases}e^{\frac{1}{1-x^{2}-y^{2}}} & \text { if }(x, y) \in \Omega, \\ 0 & \text { else },\end{cases}
$$

and a discretization $0=t_{1}<t_{2}<\ldots<t_{M+1}=T$ of $[0, T]$. For $k=$ $1,2, \ldots, M$, we set

$$
\begin{aligned}
& A(k, 1):=\int_{0}^{t_{k+1}}\left(\int_{\Omega} S_{H}(\tau, s) \Delta \psi(s) d s\right) d \tau, \\
& A(k, 2):=\int_{0}^{t_{k+1}}\left(\int_{\Omega} I_{H}(\tau, s) \Delta \psi(s) d s\right) d \tau,
\end{aligned}
$$


and

$$
\begin{aligned}
B(k) & :=\int_{\Omega}\left(S_{H}\left(t_{k+1}, s\right)+I_{H}\left(t_{k+1}, s\right)\right) \psi(s) d s-\int_{\Omega}\left(S_{H}(0, s)+I_{H}(0, s)\right) \psi(s) d s \\
& +\left(\gamma+b_{H}\right) \int_{0}^{t_{k+1}}\left(\int_{\Omega} I_{H}(\tau, s) \psi(s) d s\right) d \tau-b_{H} \int_{0}^{t_{k+1}}\left(\int_{\Omega}\left(1-S_{H}(\tau, s)\right) \psi(s) d s\right) d \tau .
\end{aligned}
$$

Then, we obtain the following approximation of (23):

$$
A d=B, \text { where } d:=\left(d_{1}, d_{2}\right)^{T} .
$$

We solve this system by the standard least squares method based upon $Q R$ factorization to obtain the parameters approximations $d_{1}^{a}$ and $d_{2}^{a}$.

The results are summarized in Table 2 where $\lambda$ is the relative error between $\left(S_{H}, I_{H}\right)$ and $\left(S_{H}^{\text {meas }}, I_{H}^{\text {meas }}\right)$, while $r e$ is the relative error between $\left(d_{1}, d_{2}\right)$ and $\left(d_{1}^{a}, d_{2}^{a}\right)$.

\begin{tabular}{c|c|c|c|c}
$\lambda$ & $1 \%$ & $5 \%$ & $10 \%$ & $20 \%$ \\
\hline$d_{1}^{a}$ & 0.1019 & 0.1011 & 0.0836 & 0.1153 \\
$d_{2}^{a}$ & 0.0186 & 0.0159 & 0.0102 & 0.0191 \\
$r e$ & 0.0880 & 0.0595 & 0.1636 & 0.1773 \\
\hline
\end{tabular}

Table 2: Calculation results and errors

One can see that the method gives good approximations of $d_{1}$ and $d_{2}$. Moreover, this method also works with a strong noise.

\section{Conclusion}

In this paper, we have investigated an identifiability method applied up to now to ordinary differential equations systems to partial differential equations systems. Furthermore, some generalization results on the $L^{2}$ framework had been obtained. This method based on algebra tools had proved to provide interesting relations after regularizing them in using the distribution theory. As shown on the two examples describing the chikungunya disease, these relations permit to provide identifiability results. Furthermore, a numerical method deduced from the differential relations had been proposed and permitted to give a first initial guess of the unknown parameters. Future works concern the exploration of the proposed method to parameters in $L^{p}$ and for other class of systems. 


\section{Appendix}

In this appendix we give the proof of Theorem 4.1. To this end it suffices to show that (3) is satisfied so that a local solution $U$ exists and then to show that $U$ is non negative and satisfies (4).

We restrict ourselves to the case $r \in C^{1}([0,+\infty) ; C(\bar{\Omega}))$. The case $r$ piecewise differentiable in time is a simple adaptation by considering only compact time intervals on which $r$ is $C^{1}$. Thus, since we assume $(\mathcal{H})$ satisfied, in all the sequel we have

$$
r:=\frac{L_{m}}{A_{m}} \in C^{1}([0, \infty) ; C(\bar{\Omega})) .
$$

Set $\eta \in(3 / 4,1)$. The domain of the fractional power $B^{\eta}$ of $B$ is given by (see theorem 16.7 of [35]):

$$
D\left(B^{\eta}\right)=\left\{\left(u_{1}, u_{2}, u_{3}\right)^{T} ; u_{1} \in \mathcal{C}, u_{2}, u_{3} \in H_{N}^{2 \eta}(\Omega)\right\}
$$

and there exists $c>0$ such that for $i=1,2$

$$
\frac{1}{c}\|f\|_{H_{N}^{2 \eta}(\Omega)} \leq\left\|B_{i}^{\eta} f\right\|_{L^{2}(\Omega)} \leq c\|f\|_{H_{N}^{2 \eta}(\Omega)},
$$

for all $f \in D\left(B_{i}^{\eta}\right)$. Moreover, since $2 \eta>1$ and $\Omega$ is bounded, from Sobolev embedding theorem, we have

$$
H_{N}^{2 \eta}(\Omega) \subset C(\bar{\Omega}) \subset L^{\infty}(\Omega) \subset L^{2}(\Omega),
$$

with continuous embedding.

First step. Existence and uniqueness of a local solution.

In all the sequel, $C$ will denote a positive constant whose value can change from line to line. Set $U:=\left(u_{1}, u_{2}, u_{3}\right) \in D\left(B^{\eta}\right), V:=\left(v_{1}, v_{2}, v_{3}\right) \in D\left(B^{\eta}\right)$, $T>0$ and $s, t \in[0, T]$. We have

$$
\begin{aligned}
\|G(t, U)-G(s, \tilde{U})\|_{2} \leq & \|-\left(s_{L} r(t)+\beta_{m} u_{3}-1\right) u_{1}+\beta_{m} u_{3} \\
& +\left(s_{L} r(s)+\beta_{m} v_{3}-1\right) \tilde{u}_{1}-\beta_{m} v_{3} \|_{L^{2}} \\
& +\beta_{H}\left\|u_{1} u_{3}-v_{1} v_{3}\right\|_{L^{2}}+\beta_{H}\left\|u_{1} u_{2}-v_{1} v_{2}\right\|_{L^{2}} .
\end{aligned}
$$


From (25) and (26), we deduce

$$
\begin{aligned}
\left\|u_{1} u_{2}-v_{1} v_{2}\right\|_{L^{2}} & \leq\left\|u_{1} u_{2}-u_{1} v_{2}+u_{1} v_{2}-v_{1} v_{2}\right\|_{L^{2}} \\
& \leq\left\|u_{1}\left(u_{2}-v_{2}\right)\right\|_{L^{2}}+\left\|\left(u_{1}-v_{1}\right) v_{2}\right\|_{L^{2}} \\
& \leq\left\|u_{1}\right\|_{\infty}\left\|u_{2}-v_{2}\right\|_{L^{2}}+\left\|v_{2}\right\|_{\infty}\left\|u_{1}-v_{1}\right\|_{L^{2}} \\
& \leq C\left\|u_{1}\right\|_{H^{2 \eta}}\left\|u_{2}-v_{2}\right\|_{L^{2}}+\left\|v_{2}\right\|_{H^{2 \eta}}\left\|u_{1}-v_{1}\right\|_{L^{2}} \\
& \leq C\left(\left\|B^{\eta} U\right\|_{L^{2}}+\left\|B^{\eta} V\right\|_{L^{2}}\right)\|U-V\|_{L^{2}} .
\end{aligned}
$$

In the same way, we also have

$$
\left\|u_{1} u_{3}-v_{1} v_{3}\right\|_{L^{2}} \leq C\left(\left\|B^{\eta} U\right\|_{L^{2}}+\left\|B^{\eta} V\right\|_{L^{2}}\right)\|U-V\|_{L^{2}} .
$$

Moreover, since $r \in C^{1}([0, \infty) ; C(\bar{\Omega}))$, arguing as previously we obtain

$$
\begin{aligned}
\|-( & \left.s_{L} r(t)+\beta_{m} u_{3}-1\right) u_{1}+\beta_{m} u_{3}+\left(s_{L} r(s)+\beta_{m} v_{3}-1\right) v_{1}-\beta_{m} v_{3} \|_{L^{2}} \\
\leq & \left(s_{L}\|r\|_{\infty} \|+1\right)\left\|u_{1}-v_{1}\right\|_{L^{2}}+s_{L}\left\|r^{\prime}\right\|_{\infty}\left\|u_{1}\right\|_{L^{2}}|t-s| \\
& \quad+\beta_{m}\left\|u_{1} u_{3}-v_{1} v_{3}\right\|_{L^{2}}+\beta_{m}\left\|u_{3}-v_{3}\right\|_{L^{2}} \\
\leq & C\left(\left\|B^{\eta}(U-V)\right\|_{L^{2}}+\left(\left\|B^{\eta} U\right\|_{L^{2}}+\left\|B^{\eta} V\right\|_{L^{2}}+1\right)\left(|t-s|+\|U-V\|_{2}\right) .\right.
\end{aligned}
$$

From inequalities (27), (28), (29) and (30), we get (3).

Set $U_{0} \in X_{+}$, where $X_{+}$is given by (19). From the first step and Theorem 4.4 of [35], we obtain that there exists $T_{U_{0}}>0$ such that (18)-(b) admits a unique local solution:

$$
U:=\left(u_{1}, u_{2}, u_{3}\right) \in C\left(\left[0, T_{U_{0}}\right] ; D(B)\right) \cap C^{1}\left(\left(0, T_{U_{0}}\right] ; X\right) .
$$

Second step. Non negativity of the local solution.

This proof is quite standard and can be found in [35] (e.g. page 197), we set it here for the reader convenience. Let $\chi(f)$ be a cutoff function defined by $\chi(f)=0$ for $f \in(-\infty, 0)$ and $\chi(f)=f$ for $f \in[0,+\infty)$. We set

$\tilde{G}(t, \tilde{U}):=\left(-\left(s_{L} r(t)+\beta_{m} \tilde{u}_{3}-1\right) \tilde{u}_{1}+\beta_{m} \tilde{u}_{3},-\beta_{H} \chi\left(\tilde{u}_{1}\right) \tilde{u}_{3}+b_{H}, \beta_{H} \chi\left(\tilde{u}_{1}\right) \tilde{u}_{2}\right)$.

Then, we consider the following problem

$$
\left\{\begin{aligned}
\partial_{t} \tilde{U}+B \tilde{U} & =\tilde{G}(t, \tilde{U}), \quad \text { in }(0, \infty), \\
\tilde{U}(0) & =U_{0} .
\end{aligned}\right.
$$


Using similar arguments as in step 1 , one can prove that there exists $\tilde{T}_{U_{0}}$ such that (32) admits a unique local solution

$$
\tilde{U}:=\left(\tilde{u}_{1}, \tilde{u}_{2}, \tilde{u}_{3}\right) \in C\left(\left[0, \tilde{T}_{U_{0}}\right] ; D(B)\right) \cap C^{1}\left(\left(0, \tilde{T}_{U_{0}}\right] ; X\right) .
$$

Consider $H(s):=\frac{1}{2} s^{2}$ for $s \in(-\infty, 0)$ and $H(s):=0$ for $s \in[0,+\infty)$. This function satisfies

$$
H(s) \geq 0, H^{\prime}(s) \leq 0 \quad \text { and } \quad H^{\prime}(s) s \geq 0, H^{\prime}(s) s \leq 2 H(s)
$$

We set

$$
\phi_{1}(t):=\int_{\Omega} H\left(\tilde{u}_{2}(t, x)\right) d x
$$

Since $x \mapsto H\left(\tilde{u}_{2}(t, x)\right)$ is continuous for all $t \in\left(0, \tilde{T}_{U_{0}}\right]$, if $\phi_{1} \equiv 0$ then $\tilde{u}_{2} \geq 0$. Since $\phi_{1}$ is continuously differentiable and from (32), we have:

$$
\begin{aligned}
\phi_{1}^{\prime}(t)= & \int_{\Omega} H^{\prime}\left(\tilde{u}_{2}(t, x)\right) \partial_{t} \tilde{u}_{2}(t, x) d x \\
= & d_{1} \int_{\Omega} H^{\prime}\left(\tilde{u}_{2}\right) \Delta \tilde{u}_{2} d x+\int_{\Omega} H^{\prime}\left(\tilde{u}_{2}\right)\left(b_{H}-\left(b_{H}+\beta_{H} \chi\left(\tilde{u}_{1}\right)\right) \tilde{u}_{2}\right) d x \\
& -b_{H} \int_{\Omega} H^{\prime}\left(\tilde{u}_{2}\right) \tilde{u}_{2} d x-\beta_{H} \int_{\Omega} H^{\prime}\left(\tilde{u}_{2}\right) \tilde{u}_{2} \chi\left(\tilde{u}_{1}\right) d x .
\end{aligned}
$$

Since $\partial_{\nu} \tilde{u}_{2}=0$ on $\partial \Omega$, we get

$$
\int_{\Omega} H^{\prime}\left(\tilde{u}_{2}\right) \Delta \tilde{u}_{2} d x=-\int_{\Omega} \nabla\left(H^{\prime}\left(\tilde{u}_{2}\right)\right) . \nabla \tilde{u}_{2} d x=-\int_{\Omega}\left|\nabla \tilde{u}_{2}\right|^{2} d x \leq 0 .
$$

Owing to (33), one gets that $\int_{\Omega} H^{\prime}\left(\tilde{u}_{2}\right) d x \leq 0, \int_{\Omega} H^{\prime}\left(\tilde{u}_{2}\right) \tilde{u}_{2} d x \geq 0$ and consequently that $\phi_{1}^{\prime}(t) \leq 0$. Thus, for any $t \in\left[0, \tilde{T}_{U_{0}}\right], 0 \leq \phi_{1}(t) \leq \phi_{1}(0)$. Since $U_{0} \in X_{+}, \tilde{u}_{0} \geq 0$ and so $\phi_{1}(0)=0$. We deduce that $\tilde{u}_{2} \geq 0$ in $\left[0, \tilde{T}_{U_{0}}\right]$.

In the same way, we obtain that $\tilde{u}_{1} \geq 0$ and $\tilde{u}_{3} \geq 0$ in $\left[0, \tilde{T}_{U_{0}}\right]$. Set $T:=$ $\min \left(T_{U_{0}}, \tilde{T}_{U_{0}}\right)$. Since $\tilde{u}_{1} \geq 0$ in $[0, T]$, we deduce from the definition (31) of $\tilde{G}$ that $\tilde{G}(\tilde{U}(t))=G(U(t))$ in $[0, T]$. By uniqueness of the solution of (18)-(b), this leads to $\tilde{U}=U$ in $[0, T]$. Thus, $u_{1}, u_{2}, u_{3} \geq 0$ in $[0, T]$.

Third step. Global non negative solution.

To prove that the solution is global, it suffices to show that the inequality (4) is satisfied. 
1. Let us prove first that $u_{1}$ is bounded. Suppose that there exists $t$ such that $u_{1}(t, x)>1$ and consider

$$
t_{1}:=\inf \left\{t \mid u_{1}(t, x)=1 \text { and } u_{1}(t+\varepsilon, x)>1, \forall \varepsilon>0\right\},
$$

then

$$
\forall t \in\left[0, t_{1}\right], \quad u_{1}(t, x) \leq 1, u_{1}\left(t_{1}, x\right)=1
$$

Since

$$
\begin{aligned}
\partial_{t} u_{1}\left(t_{1}, x\right) & \left.=-\left(s_{L} \frac{\zeta_{2}\left(t_{1}, x\right)}{\zeta_{3}\left(t_{1}, x\right)}+\beta_{m} u_{3}\left(t_{1}, x\right)\right) u_{1}\left(t_{1}, x\right)+\beta_{m} u_{3}\left(t_{1}, x\right)\right) \\
& =-s_{L} \frac{\zeta_{2}\left(t_{1}, x\right)}{\zeta_{3}\left(t_{1}, x\right)} \leq 0 .
\end{aligned}
$$

If $\partial_{t} u_{1}\left(t_{1}, x\right)=0$, then $\zeta_{2}\left(t_{1}, x\right)=0$ so $\partial_{t} \zeta_{2}\left(t_{1}, x\right)=s \zeta_{1}\left(t_{1}, x\right) \geq 0$. Recall that $\zeta_{3}\left(t_{1}, x\right) \neq 0$.

- $\partial_{t} \zeta_{2}\left(t_{1}, x\right)>0$, by continuity, there exists $\delta_{1}$ such that $\zeta_{2}(t, x)$ is strictly increasing on $\left(t_{1}-\delta_{1}, t_{1}+\delta_{1}\right)$, then $\zeta_{2}(t, x)<\zeta_{2}\left(t_{1}, x\right)=$ $0, t \in\left(t_{1}-\delta_{1}, t_{1}\right)$, which contradicts the positivity of $\zeta_{2}$.

- $\partial_{t} \zeta_{2}\left(t_{1}, x\right)=0$, then $\zeta_{1}\left(t_{1}, x\right)=0$ and $\partial_{t} \zeta_{1}\left(t_{1}, x\right)=b \zeta_{3}\left(t_{1}, x\right)>0$, so by continuity, there exists $\delta_{2}$ such that $\zeta_{1}(t, x)$ is strictly increasing on $\left(t_{1}-\delta_{2}, t_{1}+\delta_{2}\right)$, then $\zeta_{1}(t, x)<\zeta_{1}\left(t_{1}, x\right)=0, t \in\left(t_{1}-\delta_{2}, t_{1}\right)$, which contradicts the positivity of $\zeta_{1}$.

So we have $\partial_{t} u_{1}\left(t_{1}, x\right)<0$, by continuity, there exists $\delta_{3}$ such that $u_{1}(t, x)$ is strictly decreasing on $\left(t_{1}-\delta_{3}, t_{1}+\delta_{3}\right)$, then

$$
u_{1}\left(t_{1}-\delta_{3}, x\right)>u_{1}(t, x)>u_{1}\left(t_{1}, x\right), t \in\left(t_{1}-\delta_{3}, t_{1}\right) .
$$

Since $u_{1}(t, x)>u_{1}\left(t_{1}, x\right)=1, t \in\left(t_{1}-\delta_{3}, t_{1}\right)$, which contradicts the definition of $t_{1}$. So we obtain

$$
\forall t \in[0, T], \quad\left\|u_{1}(t)\right\|_{\infty} \leq 1
$$

2. Let $t \in[0, T]$. Multiplying $\partial_{t} u_{2}=-\left(b_{H}+\beta_{H} u_{1}\right) u_{2}+b_{H}+d_{1} \Delta u_{2}$ by $u_{2}$ and integrating by parts over $\Omega$, we get

$$
\begin{aligned}
\frac{1}{2} \frac{d}{d t}\left\|u_{2}(t)\right\|_{L^{2}}^{2}= & -b_{H}\left\|u_{2}(t)\right\|_{L^{2}}^{2}-\beta_{H} \int_{\Omega} u_{1}(t) u_{2}^{2}(t) d x+\int_{\Omega} b_{H} u_{2}(t) d x \\
& -d_{1} \int_{\Omega}\left|\nabla u_{2}(t)\right|^{2} d x .
\end{aligned}
$$


Since $u_{1} \geq 0, d_{1}>0$ and $b_{H} u_{2}(t) \leq \frac{b_{H}}{2}+\frac{b_{H}}{2} u_{2}^{2}(t)$, we obtain

$$
\frac{d}{d t}\left\|u_{2}(t)\right\|_{L^{2}}^{2}+b_{H}\left\|u_{2}(t)\right\|_{L^{2}}^{2} \leq b_{H}|\Omega|
$$

Solving this differential inequality leads to

$$
\forall t \in[0, T], \quad\left\|u_{2}(t)\right\|_{L^{2}}^{2} \leq C .
$$

3. In the same way, from (34), we obtain that $u_{3}$ satisfies

$$
\begin{aligned}
\frac{1}{2} \frac{d}{d t}\left\|u_{3}(t)\right\|_{L^{2}}^{2} & \leq \beta_{H} \int_{\Omega} u_{1} u_{2} u_{3} d x-\left(\gamma+b_{H}\right)\left\|u_{3}(t)\right\|_{L^{2}}^{2} \\
& \leq \beta_{H} \int_{\Omega} u_{2} u_{3} d x-\left(\gamma+b_{H}\right)\left\|u_{3}(t)\right\|_{L^{2}}^{2} .
\end{aligned}
$$

From Cauchy-Schwarz inequality and Young's inequality, for any $\varepsilon>0$, we get

$$
\begin{aligned}
\frac{1}{2} \frac{d}{d t}\left\|u_{3}(t)\right\|_{L^{2}}^{2} d x & \leq \beta_{H}\left\|u_{2}(t)\right\|_{L^{2}}\left\|u_{3}(t)\right\|_{L^{2}}-\left(\gamma+b_{H}\right)\left\|u_{3}(t)\right\|_{L^{2}}^{2} \\
& \leq \frac{\beta_{H}}{2 \varepsilon}\left\|u_{2}(t)\right\|_{L^{2}}^{2}+\frac{\beta_{H} \varepsilon}{2}\left\|u_{3}(t)\right\|_{L^{2}}^{2}-\left(\gamma+b_{H}\right)\left\|u_{3}(t)\right\|_{L^{2}}^{2} \\
& \leq \frac{\beta_{H} C_{1}}{4 \varepsilon}+\left(\beta_{H} \varepsilon-\gamma-b_{H}\right) \int_{\Omega} u_{3}^{2} d x .
\end{aligned}
$$

Taking $\varepsilon:=\left(\gamma+b_{H}\right) / \beta_{H}$, from (35), we obtain

$$
\frac{d}{d t}\left\|u_{3}(t)\right\|_{L^{2}}^{2} d x+\left(\gamma+b_{H}\right)\left\|u_{3}(t)\right\|_{L^{2}}^{2} \leq C .
$$

Solving this differential inequality leads to

$$
\forall t \in[0, T], \quad\left\|u_{3}(t)\right\|_{L^{2}}^{2} \leq C .
$$

From (34), (35) and (36) we obtain that $U$ satisfies (4), which leads to the result.

\section{References}

[1] W. Abid, R. Yafia, M.A. Aziz-Alaoui, H. Bouhafa, A. Abichou, Diffusion driven instability and Hopf bifurcation in spatial predator-prey model on a circular domain, Applied Mathematics and Computation, 260 (2015), 292313. 
[2] M. Andrle, A. El Badia, On an inverse source problem for the heat equation. Application to a pollution detection problem, Inverse Prob. Sci. Eng. 23 (3) (2015), 389-412.

[3] F. Boulier, E. Cheb-Terrab. DifferentialAlgebra. Package of MapleSoft MAPLE standard library since MAPLE 14 (2008).

[4] F. Boulier, F. Lemaire, M. Rosenkranz, R. Ushirobira, N. Verdière, On Symbolic Approaches to Integro-Differential Equations. Chapter in Algebraic and Symbolic Computation Methods in Dynamical Systems, Springer, 2017.

[5] A. P. Calderón, On an inverse boundary value problem. Seminar on Numerical Analysis and its Applications to Continuum Physics (Rio de Janeiro, 1980), pp. 65-73, Soc. Brasil. Mat., Rio de Janeiro, 1980.

[6] A. Coronel, L. Friz, I. Hess, M. Zegarra, On the existence and uniqueness of an inverse problem in epidemiology, Applicable Analysis, Published Online: 26 Apr 2019, DOI: 10.1080/00036811.2019.1608964.

[7] M. Darbas, J. Heleine, S. Lohrengel, Sensitivity analysis for 3D Maxwell's equations and its use in the resolution of an inverse medium problem at fixed frequency, Inverse Problems in Science and Engineering, Published Online: 12 Mar 2019, 10.1080/17415977.2019.1588896.

[8] L. Denis-Vidal, G. Joly-Blanchard, C. Noiret. Some effective approaches to check identifiability of uncontrolled nonlinear systems, Mathematics and Computers in Simulation, 57 (2001), 35-44.

[9] L. Denis-Vidal, G. Joly-Blanchard, C. Noiret, M. Petitot, An algorithm to test identifiability of non-linear systems, Proc. 5th IFAC NOLCOS, St Petersburg, Russia, 7 (2001), 174-178.

[10] H. Egger, H. W. Engl, M. V. Klibanov, Global uniqueness and Hölder stability for recovering a nonlinear source term in a parabolic equation, Inverse Problems, 21(1) (2005), 271-290.

[11] M. Fliess, M. Mboup, H. Mounier, H. Sira-Ramirez, Questioning some paradigms of signal processing via concret examples, Proc. Summer School: Fast Estimation Method, Automatic Control and Signal Processing, Paris (2005). 
[12] T. Glad, Nonlinear input output relations and identifiability, Proceedings of the 31st Conference of. Decision and Control, 1992.

[13] A. Hamdi, I. Mahfoudhi, Inverse source problem based on two dimensionless dispersion-current functions in 2D evolution transport equations, $J$. Inverse Ill-Posed Probl. 24 (6) (2016), pp. 663-685.

[14] D. Janzén, M. Jirstrand, M. Chappell, N. Evans, Three novel approaches to structural identifiability analysis in mixed-effects models, Computer Methods and Programs in Biomedicine, 171 (2019), pp. 141-152.

[15] J. Lassoued, M. Mahjoub, N. Zemzemi, Stability results for the parameter identification inverse problem in cardiac electrophysiology. Inverse Problems 32 (2016), no. 11, 115002, 28 pp.

[16] L. Ljung, T. Glad, On global identifiability for arbitrary model parametrizations, Automatica, 30 (1994), pp. 265-276.

[17] A. M. Lutambi, M. A. Penny, T. Smith, N. Chitnis, Mathematical modelling of mosquito dispersal in a heterogeneous environment, Mathematical biosciences, 241 (2) (2013), pp. 198-216.

[18] H. Miao, X. Xia, A. S. Perelson, H. Wu, On identifiability of nonlinear ODE models and applications in viral dynamics, SIAM Review 53 (2011), no. 1, pp. 3-39.

[19] D. Moulay, M.A. Aziz-Alaoui, H.D. Kwon, Optimal Control of Chikungunya Disease: Larvae Reduction, Treatment and Prevention, Mathematical Biosciences and Engineering, 9(2) (2012), pp. 369-392.

[20] D. Moulay, M.A. Aziz-Alaoui, M. Cadivel, The chikungunya disease: Modeling, vector and transmission global dynamics, Mathematical Biosciences, 229(1) (2011), pp. 50-63.

[21] D. Moulay, Y. Pigné, A metapopulation model for chikungunya including populations mobility on a large-scale network, Journal of theoretical biology, 318 (2013), pp. 129-139.

[22] J. D. Murray, Mathematical Biology I: An Introduction, Springer, 2002.

[23] S. Nakagiri, Review of Japanese work of the last ten years on identifiability in distributed parameter systems, Inverse Problems, 9(2) (1993), pp. 143. 
[24] A. Perasso, B. Laroche, Y. Chitour,S. Touzeau, Identifiability analysis of an epidemiological model in a structured population, Journal of Mathematical Analysis and applications, 374(1) (2011), pp. 154-165.

[25] A. Perasso, U. Razafison, Identifiability problem for recovering the mortality rate in an age-structured population dynamics model, Inverse Probl. Sci. Eng. 24 (2016), no. 4, 711-728.

[26] H. Pohjanpalo, System identifiability based on the power series expansion of the solution, Mathematical biosciences, 41(1) (1978), pp. 21-33.

[27] S. Zhu, L. Denis-Vidal, N. Verdière, Identifiability study in a model describing the propagation of the chikungunya to the human population, Proc. MOSIM'2014, Nancy, France, 5-7 November (2014).

[28] N. Tuncer, H. Gulbudak, V. Cannataro, M. Martcheva, Structural and practical identifiability issues of immuno-epidemiological vector-host models with application to Rift Valley Fever, Bull. Math. Biol. 78 (2016), no. 9, 1796-1827.

[29] G. Uhlmann, Electrical impedance tomography and Calderón's problem, Inverse Problems 25 (2009), no. 12, 123011, 39 pp, DOI: 10.1088/0266$5611 / 25 / 12 / 123011$.

[30] S. Vajda, K.R. Godfrey, H. Rabitz, Similarity transformation approach to identifiability analysis of nonlinear compartmental models, Mathematical biosciences, 93(2) (1989), pp. 217-248.

[31] N. Verdière, L. Denis-Vidal, G. Joly-Blanchard, D. Domurado. Identifiability and estimation of pharmacokinetic parameters for the ligands of the macrophage mannose receptor, International Journal of Applied Mathematics in Computer Sciences, 15 (2005), pp. 517-526.

[32] N. Verdière, S. Zhu, L. Denis-Vidal, A distribution input-output polynomial approach for estimating parameters in nonlinear models. Application to a Chikungunya model, Journal of Computational and Applied Mathematics, Vol. 331 (2018), pp. 104-118.

[33] N. Verdière, S. Orange, A systematic approach for doing an a priori identifiability study of dynamical nonlinear models. Math. Biosci. 308 (2019), pp. 105-113. 
[34] H. Xiang, B. Liu, Solving the inverse problem of an SIS epidemic reactiondiffusion model by optimal control methods, Comput. Math. Appl. 70 (2015), no. 5, pp. 805-819.

[35] A. Yagi, Abstract Parabolic Evolution Equations and their Applications, Springer-Verlag, Berlin (2010).

[36] J. Zhang, X. Xia, C.H. Moog, Parameter identifiability of Nonlinear systems with time-delay, IEEE Trans. Automat. Control, 51(2)(2006), pp. 371-375.

[37] S. Zhu, N. Verdière, L. Denis-Vidal, D. Kateb, Identifiability analysis and parameter estimation of an epidemiologic model in a spatially continuous domain, Ecological Complexity, 34 (2018), pp. 80-88 . 